\title{
Polycomb-dependent differential chromatin compartmentalization determines gene coregulation in Arabidopsis
}

\author{
Ying Huang, ${ }^{1}$ Sanchari Sicar, ${ }^{1}$ Juan S. Ramirez-Prado, ${ }^{1}$ Deborah Manza-Mianza, ${ }^{1}$ \\ Javier Antunez-Sanchez, ${ }^{2}$ Rim Brik-Chaouche, ${ }^{1}$ Natalia Y. Rodriguez-Granados, ${ }^{1}$ \\ jing An, ${ }^{1}$ Catherine Bergounioux, ${ }^{1}$ Magdy M. Mahfouz, ${ }^{3}$ Heribert Hirt, ${ }^{1,3}$ \\ Martin Crespi, ${ }^{1}$ Lorenzo Concia, ${ }^{1,4}$ Fredy Barneche, ${ }^{4}$ Simon Amiard, ${ }^{5}$ Aline V. Probst, ${ }^{5}$ \\ Jose Gutierrez-Marcos, ${ }^{2}$ Federico Ariel, ${ }^{6}$ Cécile Raynaud, ${ }^{1}$ David Latrasse, ${ }^{1}$ \\ and Moussa Benhamed ${ }^{1,7,8}$ \\ ${ }^{1}$ Université Paris-Saclay, CNRS, INRAE, Univ Evry, Institute of Plant Sciences Paris-Saclay (IPS2), 91405, Orsay, France; ${ }^{2}$ School of Life \\ Science, University of Warwick, Coventry CV4 7AL, United Kingdom; ${ }^{3}$ Division of Biological and Environmental Sciences and \\ Engineering, King Abdullah University of Science and Technology, Thuwal 23955-6900, Kingdom of Saudi Arabia; ${ }^{4}$ Institut de \\ Biologie de l'Ecole Normale Supérieure (IBENS), ENS, CNRS UMR8197, INSERM U1024, PSL Research University, 75005, Paris, \\ France; ${ }^{5} G R e D$, Université Clermont Auvergne, CNRS, INSERM, BP 38, 63001, Clermont-Ferrand, France; ${ }^{6}$ Instituto de \\ Agrobiotecnología del Litoral, CONICET, Universidad Nacional del Litoral, 3000, Santa Fe, Argentina; ${ }^{7}$ Université de Paris, Institute of \\ Plant Sciences Paris-Saclay (IPS2), F-75006 Paris, France; ${ }^{8}$ Institut Universitaire de France (IUF)
}

\begin{abstract}
In animals, distant H3K27me3-marked Polycomb targets can establish physical interactions forming repressive chromatin hubs. In plants, growing evidence suggests that H3K27me3 acts directly or indirectly to regulate chromatin interactions, although how this histone modification modulates 3D chromatin architecture remains elusive. To decipher the impact of the dynamic deposition of H3K27me3 on the Arabidopsis thaliana nuclear interactome, we combined genetics, transcriptomics, and several 3D epigenomic approaches. By analyzing mutants defective for histone H3K27 methylation or demethylation, we uncovered the crucial role of this chromatin mark in short- and previously unnoticed long-range chromatin loop formation. We found that a reduction in H3K27me3 levels led to a decrease in the interactions within Polycomb-associated repressive domains. Regions with lower H3K27me3 levels in the H3K27 methyltransferase clf mutant established new interactions with regions marked with $\mathrm{H} 3 \mathrm{~K} 9 \mathrm{ac}$, a histone modification associated with active transcription, indicating that a reduction in H3K27me3 levels induces a global reconfiguration of chromatin architecture. Altogether, our results reveal that the 3D genome organization is tightly linked to reversible histone modifications that govern chromatin interactions. Consequently, nuclear organization dynamics shapes the transcriptional reprogramming during plant development and places H3K27me3 as a key feature in the coregulation of distant genes.
\end{abstract}

[Supplemental material is available for this article.]

Gene expression regulation underlying eukaryotic cell differentiation depends largely on covalent modifications of nuclear chromatin, including histone modifications (Ahmad et al. 2010). It has been shown that histone modifications alter chromatin condensation, which ultimately affects global genome topology in the nucleus, as well as the local chromatin 3D conformation, thereby modulating the accessibility of specific loci to the transcriptional machinery (Rodriguez-Granados et al. 2016; Huang et al. 2020). In the last few years, the concept of genetic information encoded in a linear sequence of nucleotides associated with histones has evolved into a more comprehensive viewpoint considering the dynamic 3D architecture of the cell nucleus (Misteli 2007). From this perspective, structural elements such as loops, domains, territories,

Corresponding authors: david.latrasse@u-psud.fr, moussa.benhamed@u-psud.fr

Article published online before print. Article, supplemental material, and publication date are at https://www.genome.org/cgi/doi/10.1101/gr.273771.120. and factories emerge as crucial functional features controlling the physical interaction between promoters and distant regulatory elements (Sutherland and Bickmore 2009), as well as the spatial organization of transcriptional hubs. Consequently, nuclear organization dynamics appear as an integrator of developmental and environmental signals (Huang et al. 2020).

The advent of innovative methods to study genome-wide 3D spatial chromatin organization from a molecular perspective, such as Hi-C, HiChIP, and ChlA-PET, made it possible to uncover both small- and large-scale genome architecture in various cell types of metazoan organisms, notably in mammals (Fullwood et al. 2009; Mifsud et al. 2015; Mumbach et al. 2016; Mishra and Hawkins 2017). This has revealed the existence of megabase-long chromatin

(c) 2021 Huang et al. This article is distributed exclusively by Cold Spring Harbor Laboratory Press for the first six months after the full-issue publication date (see https://genome.cshlp.org/site/misc/terms.xhtml). After six months, it is available under a Creative Commons License (Attribution-NonCommercial 4.0 International), as described at http://creativecommons.org/licenses/by-nc/4.0/. 
compartments comprising either active (A compartment) or inactive chromatin (B compartment) (Lieberman-Aiden et al. 2009; Fortin and Hansen 2015; Rowley et al. 2017). Furthermore, it also allowed the identification of topologically associated domains (TADs) as fundamental units of 3D genome organization. TADs are large self-interacting genomic regions (Dixon et al. 2012, 2016; Rocha et al. 2015; Gonzalez-Sandoval and Gasser 2016), which encompass genes displaying similar expression dynamics, suggesting that physical association is functionally relevant for the control of transcriptional activity (Dekker and Heard 2015). Higher-resolution studies have shown that TADs can be further subdivided into compartmental domains, which formation appears to be governed by transcription (Rao et al. 2017; Rowley et al. 2017).

Although plant and metazoan nuclei share certain commonalities, they also display radical differences (Feng et al. 2014; Grob et al. 2014; Rodriguez-Granados et al. 2016; Dong et al. 2017; Mascher et al. 2017; Huang et al. 2020). Notably, plants lack genes encoding CTCF transcription factors, which in animals are key players in the establishment of TADs and chromatin loops (de Wit et al. 2015; Guo et al. 2015). Pioneer Hi-C analyses revealed the absence of TAD-like structures in Arabidopsis thaliana (Feng et al. 2014; Grob et al. 2014; Wang et al. 2015), a phenomenon that has been attributed to the small size of its compact genome, which shows relatively homogenous transcriptional rates and a low density of noncoding regions (Rowley and Corces 2016; Rowley et al. 2017). Recent studies in plants with large and complex genomes revealed the existence of TAD-like domains (Dong et al. 2017, 2018; Liu et al. 2017; Wang et al. 2018; Concia et al. 2020). However, unlike their animal counterparts, these TAD-like structures mainly correspond to large heterochromatic compartments, suggesting that they should rather be referred to as Intergenic CONdensed Spacers (ICONS), because they are not functionally equivalent to TADs (Concia et al. 2020; Huang et al. 2020).

The existence of a large repertoire of histone modifications and variants with distinct physical properties and associated to diverse chromatin states (e.g., constitutive heterochromatin, facultative heterochromatin, euchromatin), allows the establishment of a highly precise cell-specific transcriptional landscape in response to environmental and developmental stimuli (Kouzarides 2007; Eichten et al. 2014). The numerous changing environmental signals perceived by the cell require a pertinent response, which must be accurately modulated with time (Gambino and Pantaleo 2017). Thus, reversible and dynamic histone modifications permit the induction and repression of the appropriate subsets of genes under certain conditions (Pfluger and Wagner 2007). In agreement, protein complexes and enzymes with opposite activity upon histone covalent modifications continuously shape the chromatin environment of target genes. Thus, the so-called writers govern the deposition of a specific covalent histone modification, whereas erasers participate in their removal (Mach 2018). A third group of proteins called readers, recognizes specific histone modifications and induces downstream processes through diverse mechanisms, including chromatin compaction, remodeling, the recruitment of secondary chromatin modifiers, transcription factors (TFs), and the DNA damage repair machinery, among others (Yun et al. 2011).

The trimethylation of the lysine 27 of histone H3 (H3K27me3) is a repressive covalent histone modification resulting from the activity of Polycomb repressive complexes (PRCs), formed by the combination of multiple Polycomb group (PcG) proteins (Grossniklaus and Paro 2014). In both animals and plants, PRCs are classified into PRC1 and PRC2, in which PRC2 deposits H3K27me3 on its tar- gets, whereas PRC1 recognizes these histone modifications and stabilizes PRC2-mediated repression. There are three SET-domain proteins with $\mathrm{H} 3 \mathrm{~K} 27 \mathrm{me} 3$ methyltransferase activity in $A$. thaliana: SWINGER (SWN), CURLY LEAF (CLF), and MEDEA (MEA) (Mozgova et al. 2015). The PcGs activity in this plant model is counteracted by four Jumonji (JMJ)-type proteins, which perform the active demethylation of H3K27me3 and determine spatial boundaries for this histone modification, restricting its spreading and the consequent formation of repressive chromatin domains (Yan et al. 2018). Among them, RELATIVE OF EARLY FLOWERING 6 (REF6) has been reported to promote flowering through the activation of the flower integrators FLOWERING LOCUS T $(F T)$ and SUPPRESSOR OF OVEREXPRESSION OF CO 1 (SOC1) (Noh et al. 2004; Hou et al. 2014). Moreover, the ref6 mutation partially complements the developmental phenotype and the decreased fertility caused by the $c l f$ mutation (Lu et al. 2011). Consistently, the REF6 overexpressing plants share several of the developmental phenotypes of $c l f$, highlighting the antagonistic function between this protein and the PRC2 complex (Lu et al. 2011).

Growing evidence indicates that H3K27me3 plays an important role in the spatial organization of chromatin in eukaryotes, a phenomenon that has been associated with its repressive role in transcription. For instance, it has been shown in animals that Polycomb targets marked with H3K27me3 can establish interactions between them, forming repressive chromatin hubs dependent on PRCs and insulator proteins (Lanzuolo et al. 2007; Cheutin and Cavalli 2014). PRCs have also been shown to regulate the maintenance of pluripotency in mammalian embryonic stem cells through the organization of interaction networks (Schoenfelder et al. 2015), and H3K27me3 levels have been associated with the Drosophila's genome subdivision into TADs (Francis et al. 2004; Eskeland et al. 2010; Williamson et al. 2012; Joshi et al. 2015; Boettiger et al. 2016; El-Sharnouby et al. 2017; Kundu et al. 2017; McLaughlin et al. 2019). In plants, several studies suggested that this covalent histone modification is a key contributor to chromatin topology. A Hi-C approach served to propose that the local interaction of $\mathrm{H} 3 \mathrm{~K} 27 \mathrm{me} 3$ domains is reduced in the Arabidopsis clf/swn double mutant background (Feng et al. 2014). Besides, it has been shown that H3K27me3 is enriched at long-distance interacting loci across the Arabidopsis genome (Liu et al. 2016a). Furthermore, in a previous study, we showed that the Drosophila HETEROCHROMATIN PROTEIN 1 (HP1) homolog in Arabidopsis LIKE-HP1 (LHP1) participates in the 3D conformation of the Arabidopsis genome (Veluchamy et al. 2016), and that its genome-wide distribution perfectly matches that of H3K27me3. LHP1 is considered a component of the plant PRC1 complex, given its capacity to recognize H3K27me3 through its chromodomain (Gaudin et al. 2001; Turck et al. 2007; Zhang et al. 2007). Therefore, H3K27me3 may directly or indirectly participate in the regulation of the dynamic interactivity of Polycomb target genes. However, several aspects of the molecular function of H3K27me3 in 3D chromatin architecture determination remain unclear. To gain a deeper understanding of the molecular role of H3K27me3, we combined cutting-edge technologies applied to the characterization of $3 \mathrm{D}$ chromatin conformation.

\section{Results}

\section{Arabidopsis chromatin organization displays a strong} compartmentalization

The first Hi-C analyses performed in Arabidopsis nuclei revealed that TADs, which are widely distributed among metazoans, are 
not obvious in this plant's genome (Liu et al. 2016a). Long-range chromatin interactions are rare in this organism and correspond almost exclusively to heterochromatic regions such as centromeres. A notable exception of this phenomenon is the KNOT, which brings together heterochromatic islands within euchromatin (Grob et al. 2014).

To determine the extent to which chromatin compartmentalization is a characteristic feature of the Arabidopsis genome, we performed immunodetection of H3K27me3 and H3K9ac, two histone posttranslational modifications associated to gene repression and activation, respectively. The immunostaining performed on somatic nuclei during interphase revealed a heterogeneous distribution of these covalent histone modifications, suggesting that distinct euchromatin and facultative heterochromatin compartments exist in Arabidopsis (Fig. 1A; Supplemental Fig. S1). Moreover, by measuring the H3K9ac and H3K27me3 signal distribution along a virtual axis arbitrarily defined in an immunostained nucleus, we observed that the corresponding peaks are anticorrelated (Fig. 1B). Given the proximity between H3K9ac and H3K27me3 marked genes along the chromosome and the resolution of the confocal microscope that is about $0.5 \mu \mathrm{m}$, this result suggests that actively transcribed and repressed genes occupy distinct compartments within the cell nucleus. To gain a deeper understanding of the compartmental organization of active and silent chromatin, we conducted HiChIP, a sensitive method for the efficient analysis of protein-centric chromosome conformation (Fig. 1C,D; Supplemental Fig. S2; Mumbach et al. 2016). H3K27me3- and H3K9ac- HiChIPs were performed in 14-d-old wild-type (WT) shoots. A minimum of 159 million read pairs were produced for each sample, and 116 million uniquely mapped read pairs were generated (Supplemental Table S1), with a high reproducibility between replicates (Supplemental Fig. S3). In parallel, ChIP-seq was performed for the same histone modifications. In contrast to traditional Hi-C (Fig. 1C,D, left panels), the HiChIP approach allows revealing interactions associated with each specific histone modification (Fig. 1C,D, right panels). Both short- and long-range interactions from a few kilobases to several megabases were detected at the site of the respective histone modifications (Supplemental Fig. S4; Supplemental Tables S2-S5). By overlapping both H3K9ac and H3K27me3 HiChIP signals, our data suggested that genes associated to the same histone modification interact with each other to form contact domains and fold the genome locally to allow the spatial segregation of transcriptionally active and silent chromatin (Fig. 1E).

\section{Shoot and root nuclei display distinct 3D chromatin architectures}

The $3 \mathrm{D}$ configuration of the nuclear genome is nonrandom and correlates with cell type-specific transcriptomes; however, the mechanisms implicated in the establishment of this configuration are poorly understood in plants. It has been recently shown that biosynthetic nonhomologous gene clusters are embedded in local 3D configurations that segregate cluster regions from the surrounding chromosome environment. Based on $\mathrm{Hi}-\mathrm{C}$ and H3K27me3 distribution in Arabidopsis, it has been proposed that genome topology differs between shoots and roots (Nützmann et al. 2020). To further decipher the relationship between H3K27me3 and 3D chromatin conformation, we performed H3K27me3- HiChIP and ChIP-seq in shoots and roots. The highresolution interaction matrix obtained by HiChIP revealed the widespread presence of interactions between genomic bins including H3K27me3-enriched genes from both tissues (Supplemental
Figs. S5, S6). A differential analysis using HOMER comparing shoots and roots served to identify the most informative loops associated with H3K27me3 (Fig. 2A), which allowed us to focus on the top shoot-specific repressive loops (SSRLs) in downstream analyses (Supplemental Table S6). We found a positive correlation between H3K27me3 levels and the strength of the detected interactions when SSRLs were visualized at different scales (Fig. 2B,C). To further support our observations, we quantified the level of this histone modification in shoots and roots on genes associated to SSRLs, and we observed that $47 \%$ of those genes are hypermethylated in shoot compared to root, confirming that this repressive histone modification is associated with chromatin loop formation and/or stability (Fig. 2D; Supplemental Table S7).

We then wondered whether gene pairs connected in SSRLs show similar changes in expression levels between shoots and roots. Using publicly available gene expression data (Liu et al. $2016 \mathrm{~b}$ ), we plotted the top SSRL gene pairs using the $\log _{2}$ (fold change shoot/root) of the first gene as the predictor and the $\log _{2}$ (fold change shoot/root) of the second gene as the response (Fig. 2E; Supplemental Fig. S7; Supplemental Table S8). This analysis revealed a significant correlation between H3K27me3 SSRL-associated loci and genes repressed in shoots (Fig. 2E). These data indicate that genes displaying specific physical contacts, and associated with H3K27me3, show similar transcriptional behavior and are likely part of common Polycomb-repressive compartments (Fig. 2E).

It has been extensively shown that $\mathrm{H} 3 \mathrm{~K} 27 \mathrm{me} 3$ works as a repressive covalent histone modification participating in the regulation of multiple developmental genes in plants (Cheng et al. 2020). Then, to better understand the impact of H3K27me3-dependent chromatin architecture on plant development, we performed an analysis of Gene Ontology (GO) terms enriched among loci grouped in SSRLs with a lower expression in shoots than in roots. We found a significant enrichment in GO categories related to root development and cell identity (Fig. 2F), hinting at a comprehensive epigenetic reprogramming of developmental pathways in different organs. Reciprocally, we also explored the root-specific repressive loops (RSRLs) and their impact on developmental outputs (Fig. 2G; Supplemental Table S9). In agreement with our observations for SSRLs, genes associated in RSRLs displayed higher H3K27me3 levels in roots than in shoots and lower expression levels in roots compared to shoots $\left(P\right.$-value $\left.=3.2 \times 10^{-55}\right)$ (Fig. 2G-K; Supplemental Fig. S7; Supplemental Tables S10, S11). In addition, the GO analysis of genes grouped in RSRLs, which are down-regulated in roots, showed enrichment in categories related to shoot development and photosynthesis (Fig. 2L), in agreement to the nonphotosynthetic nature of root tissues. These observations suggest a role of H3K27me3dependent genome topology in coordinating the transcriptional activity underlying developmental programs.

One limitation of HiChIP when using antibodies directed against a particular histone modification is that the detection of chromatin contact relies on the presence of this given histone modification at the site of interaction. Thus, the observed reduction of the strength of some interactions could merely reflect H3K27me3 depletion at loci of interest. To rule out this possibility, we performed Capture-Hi-C (C-Hi-C). This approach combines Hi$\mathrm{C}$ with the hybridization-based capture of targeted genomic regions (Supplemental Fig. S8). To this end, we generated a biotinylated RNA bait library specifically targeting 4650 regions that accumulate H3K27me3 and included a subset of loci differentially methylated between shoots and roots (Supplemental Fig. S9). The

\section{Genome Research}

www.genome.org 
A

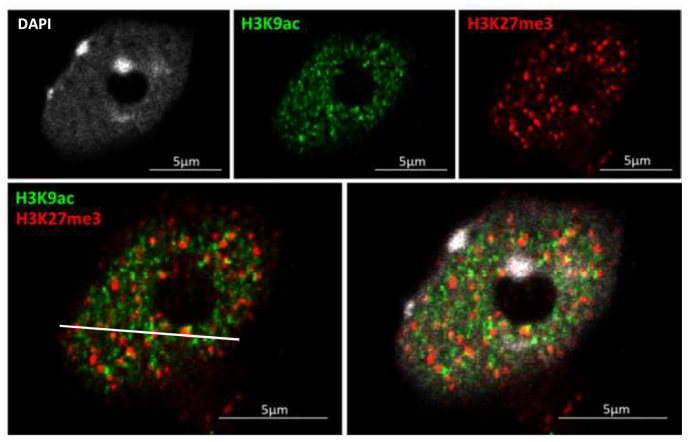

B

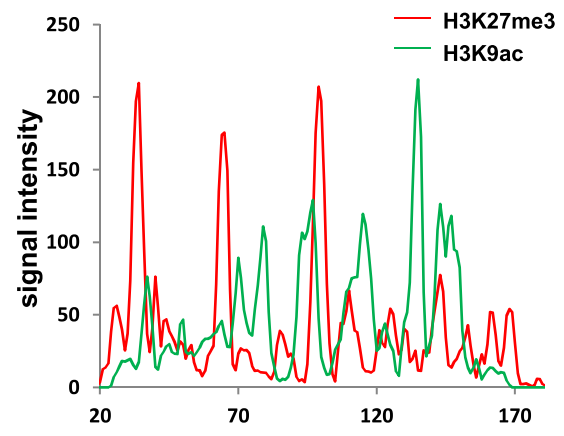

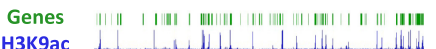

3K9ac ${ }_{4000}$ кв

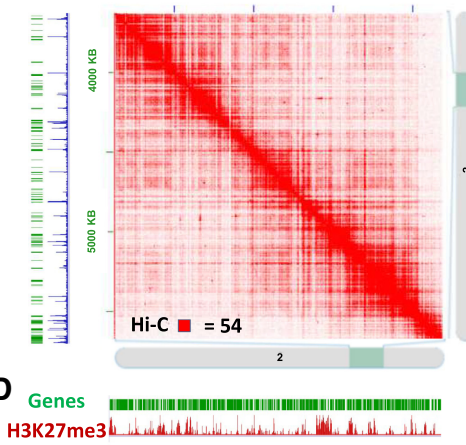

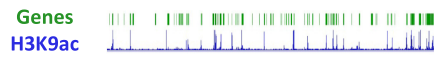

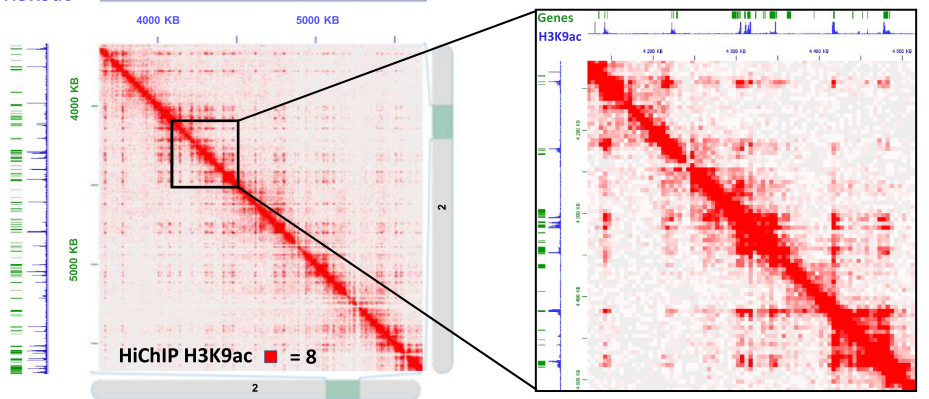

$8000 \mathrm{~KB} \quad 9000$ KB

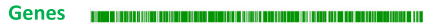

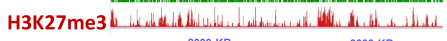
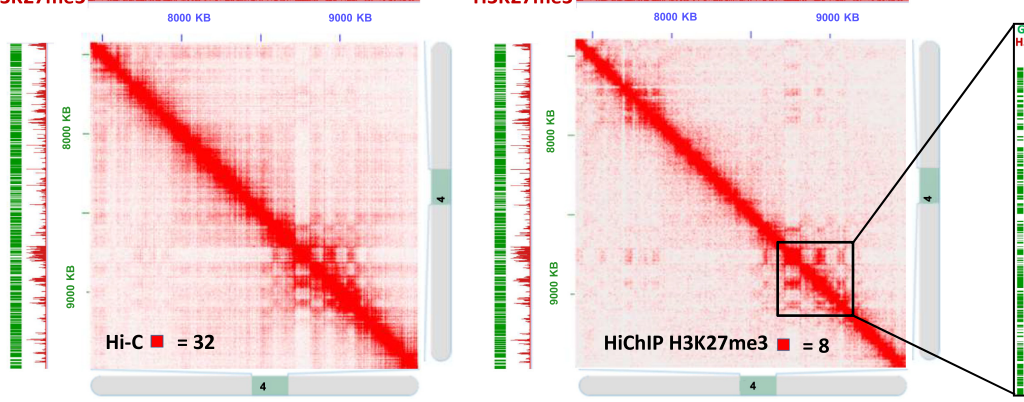

$\mathbf{E}$

$$
\overbrace{-25-18}^{-18 \text { HichIP differential interactions }}=
$$

Genes
H3K9ac
H3K27mes

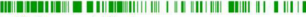

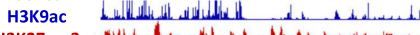

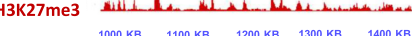

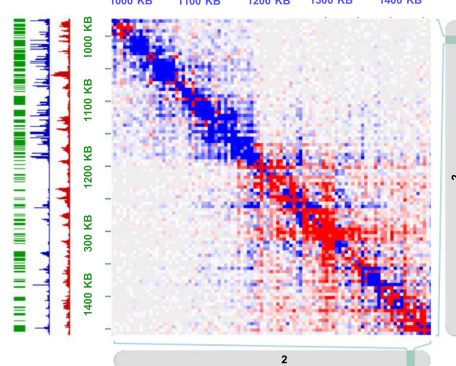

Figure 1. Arabidopsis chromatin organization displays strong compartmentalization. $(A)$ Immunofluorescence detection of H3K9ac (green) and H3K27me3 (red) histone modifications and DAPI staining (gray) in an isolated Arabidopsis nucleus. (Scale bar) $5 \mu$ m. (B) Distribution of immunofluorescence signal intensity in the nucleus. The analysis was performed along the white line shown in the merged image in $A$. ( $C$ ) Visualization of the interaction matrix of $\mathrm{Hi}-\mathrm{C}$ and HiChIP in a specific region of Chromosome 2. H3K9ac ChIP-seq signal (blue peaks) were aligned with the maps to highlight the correlation with HiChIP enriched regions as expected. (D) Visualization of the interaction matrix of Hi-C and HiChIP in a specific region of Chromosome 4. H3K27me3 ChIP-seq signal (red peaks) were aligned with the maps to highlight the correlation with HiChIP enriched regions as expected. (E) Visualization of the interaction matrix of HiChIP data of H3K9ac and H3K27me3 in a specific region of Chromosome 2. Dots showing higher (blue) and lower (red) signals in H3K9ac HiChIP compared to H3K27me3, respectively. ChIP-seq signals of H3K9ac (blue peaks) and H3K27me3 (red peaks) were aligned with the map to highlight the correlation with HiChIP enriched regions. 
A

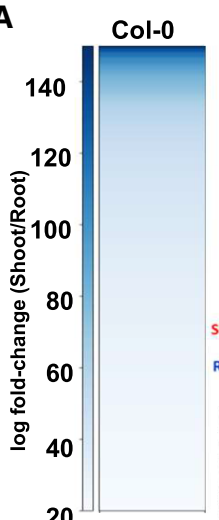

D

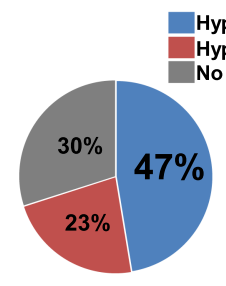

G

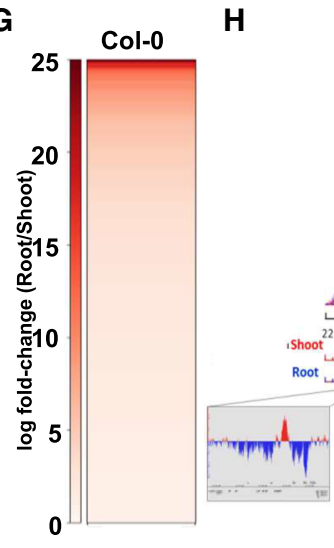

J

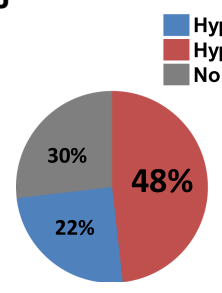

B

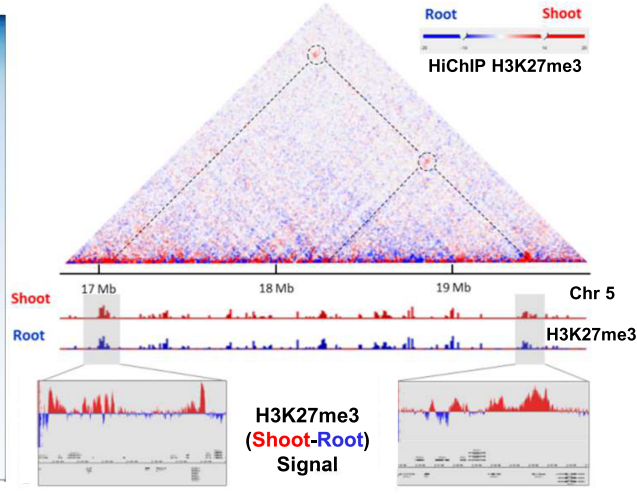

E

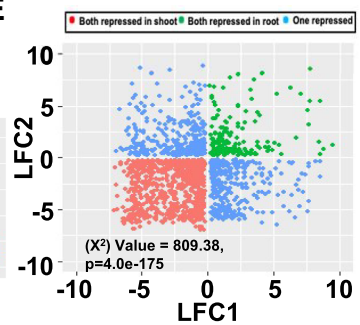

C

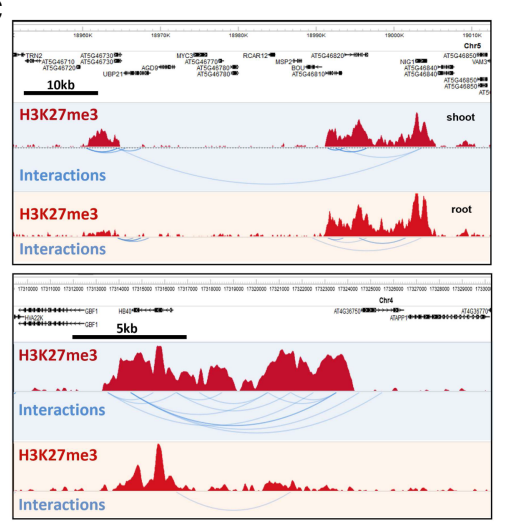

F

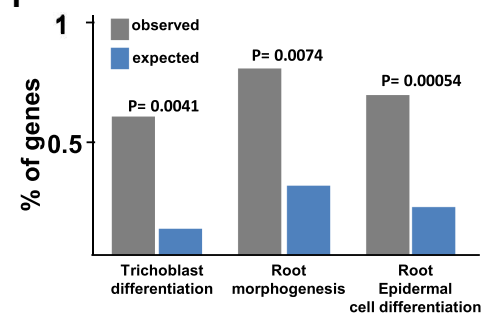

I

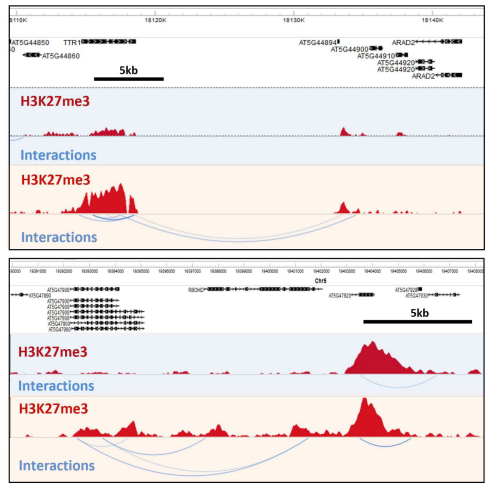

H3K27me3 (Shoot-Root) Signal

K

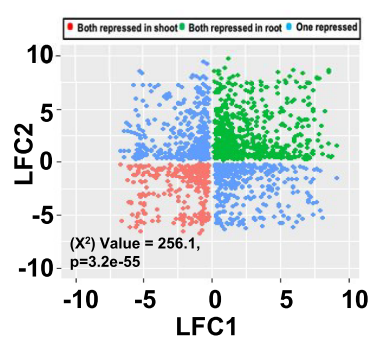

L

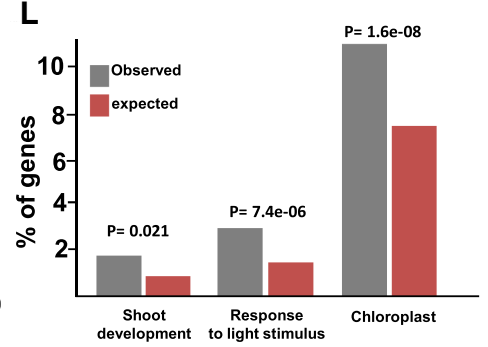

Figure 2. Shoot and root nuclei display distinct 3D chromatin architectures. (A) Heatmap showing the H3K27me3 HiChIP signal of the top shoot-specific repressive loops (SSRLs). (B) Example of long-distance SSRL on Chromosome 5. ChIP-seq signals of H3K27me3 in shoot (red peaks) and root (blue peaks) were aligned with the map and the differential analysis of both ChIP-seq signals in differentially interacting regions are highlighted (bottom panels in gray). (C) Example of short distance SSRL. H3K27me3 ChIP-seq signal is represented by red peaks and chromatin interactions signal by blue lines. (D) Analysis of H3K27me3 levels on SSRLs. The pie chart represents the percentage of the genes involved in SSRLs that are either hypermethylated in shoot or in root. The box plot shows the H3K27me3 levels in shoot or root of the $47 \%$ of shoot hypermethylated genes involved in SSRLs. (E) Scatterplot of log 2 (shoot/root gene expression fold change) for pairs of genes interacting through $\mathrm{H} 3 \mathrm{~K} 27 \mathrm{me} 3$-associated contacts in shoot. ( $F$ ) Gene Ontology enrichment analysis of the differentially expressed genes involved in SSRLs. (G) Heatmap of H3K27me3 HiChIP signal of the top root-specific repressive loops (RSRLs). (H) Example of long-distance RSRLs on Chromosome 1. ChIP-seq signals of H3K27me3 in shoot (red peaks) and root (blue peaks) were aligned with the map and the differential analysis of both ChIP-seq signals in differentially interacting regions are highlighted (bottom panels in gray). (I) Example of short distance RSRLs. (J) Analysis of H3K27me3 level over RSRLs. The pie chart represents the percentage of genes involved in RSRLs that are either hypermethylated in shoot or root. The box plot displayed the H3K27me3 levels of the $48 \%$ of root hypermethylated genes involved in RSRLs. (K) Scatterplot of log 2 (shoot/root gene expression fold change) for pairs of genes interacting through H3K27me3-associated contacts in root. (L) Gene Ontology enrichment analysis of the differentially expressed genes involved in RSRLs.

\section{Genome Research}

www.genome.org 
comparison of both Hi-C and C-Hi-C matrixes allowed us to identify a significant enrichment of chromatin contacts over our target sequences (Fig. 3A) and to obtain a high-resolution map of interactions between captured regions, as well as between captured and noncaptured regions (Fig. 3B). A differential analysis using HOMER resulted in the identification of 7676 shoot-specific loops (SSLs), of which 277 overlapped with the SSRLs previously identified by HiChIP. These data suggest that the deposition of H3K27me3 participates in the establishment of tissue-specific chromatin loops (Fig. 3C-E; Supplemental Tables S12, S13, and S8). Focusing on these SSLs identified by both methods, we found that $67 \%$ of the genes grouped in SSLs were found to be specifically repressed in shoots compared to roots (Fig. 3F). This proportion is significantly higher than the one expected by chance $(P$-value $=$ $1.61 \times 10^{-144}$ ) (Supplemental Fig. S10). On the other hand, we found through the same approach 6737 root-specific loops (RSLs), among which 231 were also found on the previously generated H3K27me3-HiChIP data analysis and defined RSRLs (Fig. 3G-I; Supplemental Tables S13, S14). Of the genes grouped in RSLs, 54\% were specifically repressed in roots compared to shoots, a proportion significantly higher than the one expected by chance $\left(P\right.$-value $\left.=2.71 \times 10^{-68}\right)$ (Fig. 3J; Supplemental Fig. S10). We also observed that $24 \%$ and $35 \%$ of the genes respectively grouped in SSLs and RSLs displayed an unexpected behavior, which could be explained by the fact that both shoots and roots correspond to complex samples of a mixture of various cell types. Altogether, our C-Hi-C data further supported that the H3K27me3 levels mediate the establishment and/or stability of repressive domains bringing together several genes in Arabidopsis and that Polycomb-related repressive compartments could play an important role in the transcriptional reprogramming underlying plant cell fate and organ development.

\section{Ectopic deposition of H3K27me3 leads to occurrence of novel chromatin repressive loops}

To decipher the impact of H3K27me3 levels on chromatin loop dynamics, we used the loss-of-function ref6-5 mutant, defective for a histone demethylase and displaying ectopic accumulation of H3K27me3 at thousands of loci (Fig. 4A; Lu et al. 2011; Yan et al. 2018; Antunez-Sanchez et al. 2020). Thus, to assess if ectopic H3K27me3 deposition could lead to the formation of new repressive compartments, we performed a C-Hi-C experiment comparing ref6-5 and WT shoots (Fig. 4B-D; Supplemental Fig. S11). A HOMER differential analysis identified the top ref6-specific repressive loops (reSLs) ranked according to the log fold change and $Z$-score (Fig. 4B; Supplemental Table S15). We observed that a large proportion of genes involved in reSLs displayed a significant increase in H3K27me3 levels in ref6-5 compared to WT $(P$-value $=4.62 \times$ $10^{-76}$ ) (Fig. 4C-E; Supplemental Fig. S12; Supplemental Table S16). To confirm this, we assessed the proportion of ref6-5 hypermethylated genes associated to repressive loops and found that $40 \%$ were associated with reSLs (Fig. 4C-E). These data suggest that a gain in H3K27me3 triggers the establishment of novel chromatin interactions (Fig. 4D). Using publicly available transcriptomic data (Antunez-Sanchez et al. 2020), we assessed whether gene pairs connected through reSLs showed similar transcriptional shifts between ref6-5 and WT. We found a significant enrichment of corepressed reSL-paired gene loci among the 546 plotted couples $\left(P\right.$-value $\left.=4 \times 10^{-10}\right)$ (Fig. 4F; Supplemental Fig. S12; Supplemental Table S17). This result indicates that genes displaying specific physical contact in ref6-5 are globally repressed, likely linked to the ec- topic accumulation $\mathrm{H} 3 \mathrm{~K} 27 \mathrm{me} 3$ and the formation of repressive chromatin loops.

\section{Reduction of H3K27me3 level induces a reconfiguration of chromatin architecture}

Considering that the ectopic deposition of H3K27me3 induces the formation of novel chromatin repressive loops, we decided to assess the impact of a reduction in H3K27me3 deposition on the interaction profile within Polycomb-related repressive domains. To this end, we used clf-29, a Polycomb Repressive Complex 2 (PRC2) histone methyltransferase mutant that displays a strong reduction on H3K27me3 levels at thousands of loci (Fig. 4A; Veluchamy et al. 2016). C-Hi-C and a differential analysis comparing clf-29 and WT shoots identified the top clf-specific disrupted loops (cSDLs) ranked according to the log fold change and $Z$-score (Fig. 5A,B; Supplemental Fig. S13; Supplemental Table S18). We observed that $33 \%$ of genes involved in cSDLs displayed a significant decrease in $\mathrm{H} 3 \mathrm{~K} 27 \mathrm{me} 3$ levels in the clf-29 mutant compared to WT $\left(P\right.$-value $=9.72 \times 10^{-52}$ ) (Fig. 5C,D; Supplemental Table S19). To confirm this observation, we assessed the proportion of clf hypomethylated genes associated to cSDLs and found that $40 \%$ of the $c l f$ hypomethylated genes are associated to a cSDL, suggesting that the loss of $\mathrm{H} 3 \mathrm{~K} 27 \mathrm{me} 3$ induced a destabilization of repressive loops in the clf-29 (Fig. 5E). To uncover the impact of chromatin architecture reorganization on transcriptional reprogramming, we analyzed the expression of gene pairs normally brought together into spatial proximity in WT shoots, which are no longer clustered in the clf-29. We observed a significant enrichment in transcriptionally activated genes $\left(P\right.$-value $\left.=4.336 \times 10^{-7}\right)$ (Fig. 5G; Supplemental Fig. S14; Supplemental Table S20), indicating that genes involved in loops lost in the clf-29 mutant tend to be up-regulated. Altogether, our results suggest that a clf-dependent decrease in H3K27me3 leads to a reconfiguration of chromatin architecture and the destabilization of H3K27me3-mediated repressive loops (Fig. 5B,F). This phenomenon can be associated to the transcriptional derepression of hundreds of genes in PRC2-related mutants.

To better understand the association between genome topology reconfiguration, H3K27me3 deposition and gene expression, we compared epigenomic and transcriptional features found in clf-29 and WT and integrated these data sets with chromatin interaction positioning (Supplemental Table S21). Our analysis revealed that interactions occur more frequently between genes with concordant transcriptional status, independently of whether they are up- or down-regulated in clf-29 compared to the WT (Fig. 5G,H). Moreover, a similar trend was observed for the epigenomic profile, because genes with concordant H3K27me3 levels are more prone to interact in common hubs (Fig. 5G,H). A higher frequency in the interaction among hypomethylated genes in clf-29 suggested a gain of novel interactions. Considering that $c l f$ hypomethylated genes showed a global transcriptional activation, we assessed in WT shoots the distribution of the H3K9ac histone modification, characteristic of active loci. Then we mapped the gained interactions determined by C-Hi-C in clf-29 throughout the genome, together with the distribution of the H3K9ac histone modification in the WT. We observed that regions losing H3K27me3 in clf-29 tend to establish novel interactions with regions marked with the active histone modification H3K9ac (Fig. 5I,J; Supplemental Figs. S15, S16; Supplemental Tables S22-S24). This suggests that a reduction in H3K27me3 levels induces a global reconfiguration 
A

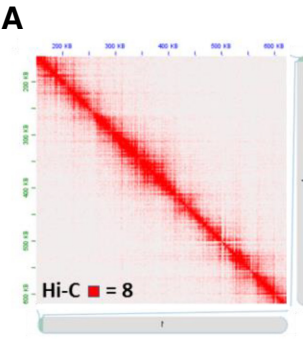

C

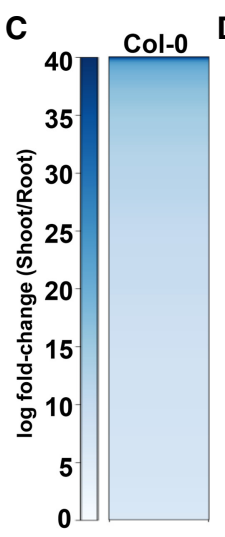

G

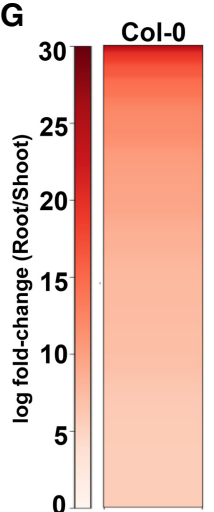

$D$

$\mathrm{H}$
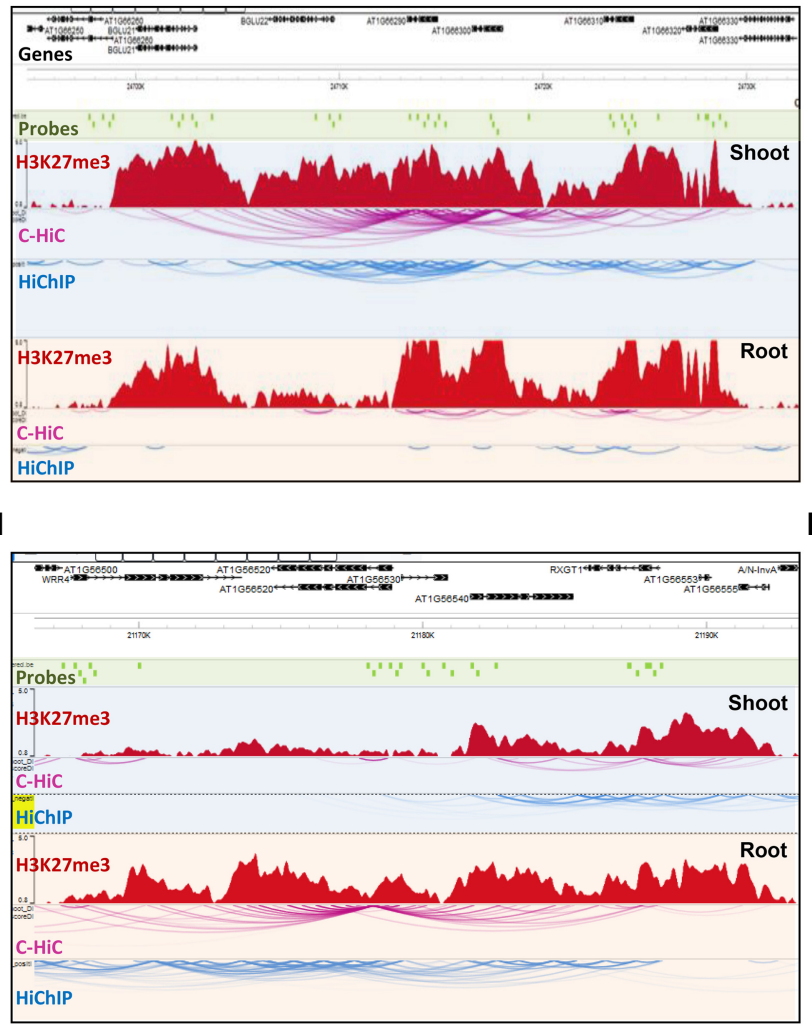

B

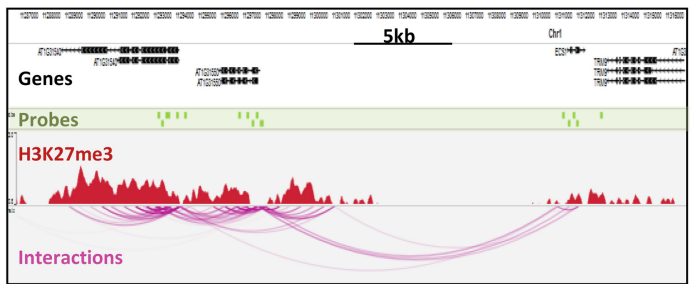

E HiChIP C-HiC

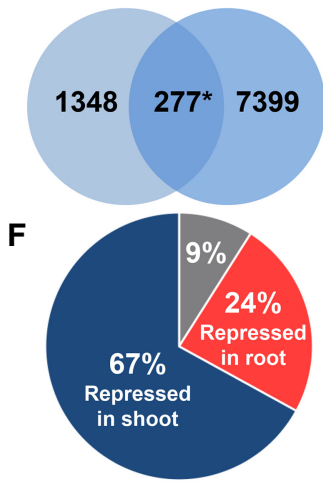

I HiChIP C-HiC

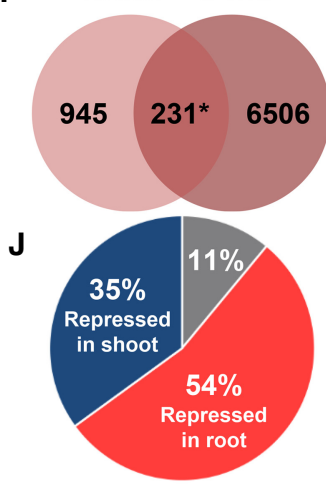

Figure 3. The levels of $\mathrm{H} 3 \mathrm{~K} 27 \mathrm{me} 3$ correlate with the stability of repressive loops. $(A)$ Visualization of the interaction matrix of $\mathrm{Hi}-\mathrm{C}$ and $\mathrm{C}-\mathrm{Hi}$ - $\mathrm{C}$ in a specific region of Chromosome 1. (B) Example of interaction analysis using C-Hi-C data showing captured regions (green bars), $\mathrm{H} 3 \mathrm{~K} 27 \mathrm{me} 3 \mathrm{ChIP}$-seq signal (red peaks), and chromatin interactions (purple lines). (C) Heatmap of C-Hi-C data showing the shoot-specific loops (SSLs). (D) Examples of the shoot-specific interacting region detected by both $\mathrm{C}-\mathrm{Hi}-\mathrm{C}$ and $\mathrm{H} 3 \mathrm{~K} 27 \mathrm{me} 3 \mathrm{HiCh}$ IP. Probes used for the $\mathrm{C}-\mathrm{Hi}-\mathrm{C}$ are represented by green bars, the $\mathrm{H} 3 \mathrm{~K} 27 \mathrm{me} 3 \mathrm{ChIP}$-seq signal by red peaks, the $\mathrm{C}-\mathrm{Hi}-\mathrm{C}$ interaction signals by purple lines, and $\mathrm{H} 3 \mathrm{~K} 27 \mathrm{me} 3 \mathrm{HiChIP}$ interaction signals by blue lines. $(E)$ Venn diagram representing overlap of loops called from HiChIP and C-Hi-C library sets. Only loops containing specific probes were selected for the comparison in HiChIP. $(F)$ Pie chart representing the proportion of genes involved in shoot-specific loops that are repressed in shoot (blue, 67\%), repressed genes in root (red, 24\%), and unchanged (gray, 9\%) among the genes involved in loops detected both with HiChIP and C-Hi-C. (G) Heatmap of C-Hi-C data showing the top root-specific loops (RSLs). $(H)$ Examples of root-specific interacting region detected by both $\mathrm{C}-\mathrm{Hi}-\mathrm{C}$ and H3K27me3 HiChIP. Probes used for the C-Hi-C are represented by green bars, the $\mathrm{H} 3 \mathrm{~K} 27 \mathrm{me} 3 \mathrm{ChIP}$-seq signal by red peaks, the $\mathrm{C}-\mathrm{Hi}-\mathrm{C}$ interaction signals by purple lines, and $\mathrm{H} 3 \mathrm{~K} 27 \mathrm{me} 3 \mathrm{HiCh} / \mathrm{P}$ interaction signals by blue lines. (I) Venn diagram representing overlap of loops called from HiChIP and C-Hi-C library sets. Only loops containing specific probes were selected for the comparison in HiChIP. (/) Pie chart representing genes involved in RSLs that are repressed in shoot (blue, 35\%), repressed genes in root (red, 54\%), and unchanged (gray, 11\%) among the genes involved in loops detected both with HiChIP and C-Hi-C.

of chromatin architecture (Fig. 5J,K), allowing genes that lost their repressive covalent histone modification to integrate transcriptionally active hubs. Altogether, our results show that the 3D genome organization is tightly linked to histone modifications that dynamically modulate the determination of chromatin domains associated with gene coregulation, thereby allowing the progression of plant developmental programs. Collectively, our data support the view that H3K27me3 acts as a key regulator of global and local facultative heterochromatin topology in plants.

\section{Discussion}

The first analyses of the 3D genome organization of Arabidopsis revealed the absence of TAD structures and the existence of an " $\mathrm{A} / \mathrm{B}$ " type compartmentalization at a local level in domains of a few tens of kilobases (Lieberman-Aiden et al. 2009; Grob et al. 2014). Constitutive heterochromatin, which contains mainly transposable element marked by specific histone modifications such as H3K9me2 and H3K27me1, is one of the major factors directing this compartmentalization. However, the contribution of

\section{Genome Research}

www.genome.org 
A

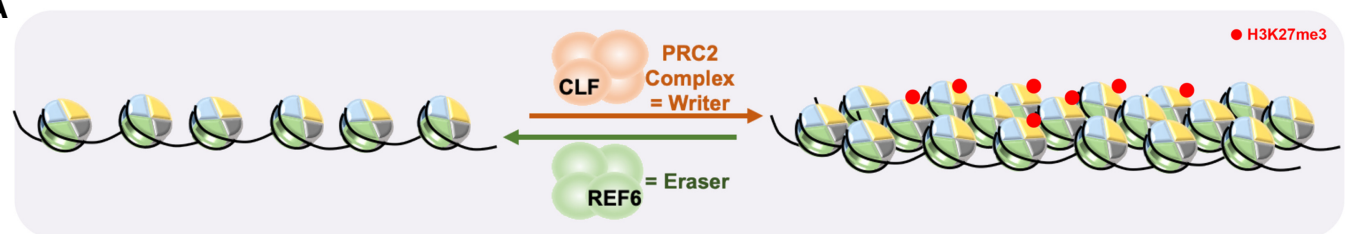

B

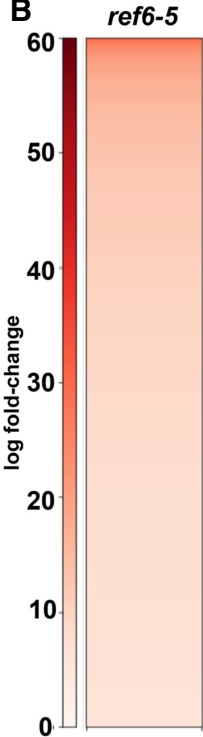

C

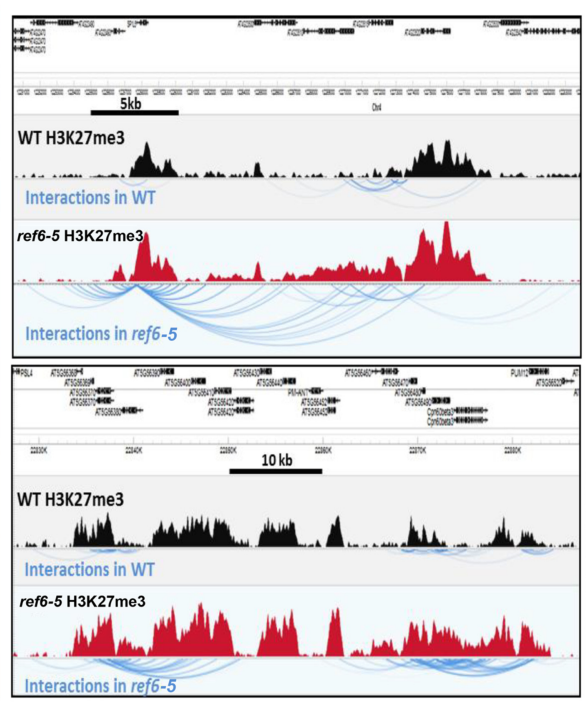

D
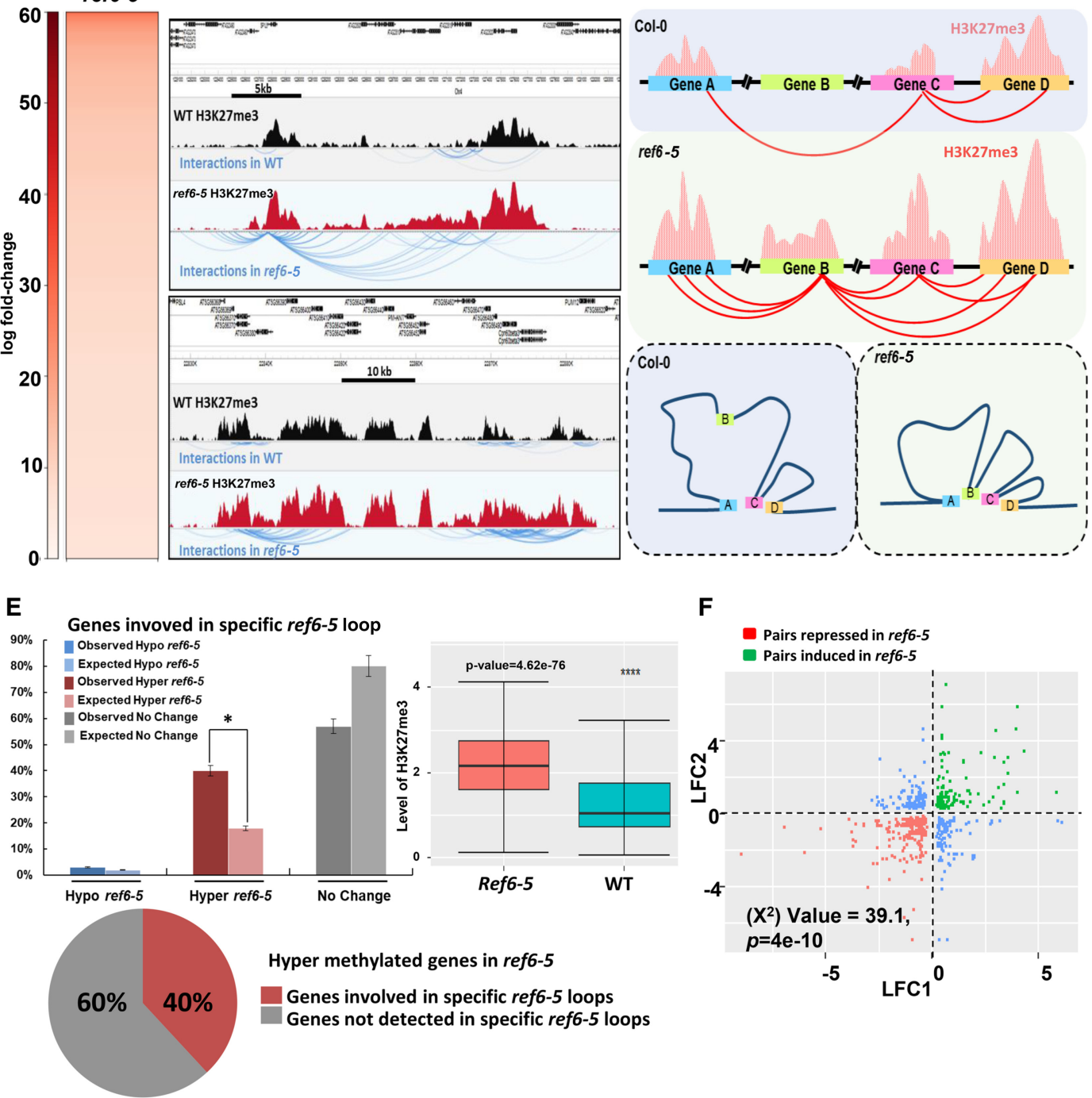

$\mathbf{F}$

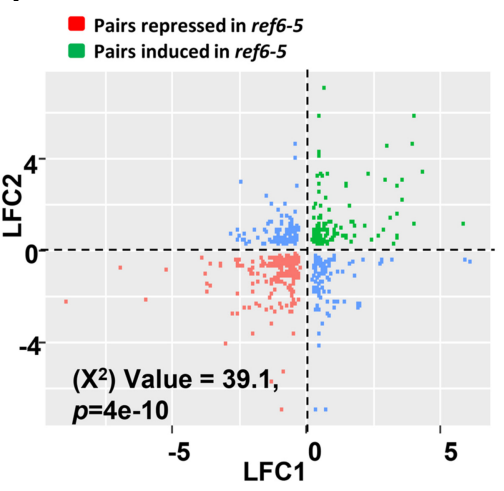

Figure 4. Ectopic deposition of $\mathrm{H} 3 \mathrm{~K} 27 \mathrm{me} 3$ leads to formation of new chromatin repressive loops. (A) Schema illustrating the antagonistic role of the PRC2 complex (involving the histone methyltransferase CLF) and the histone demethylase REF6 to control H3K27me3 homeostasis and chromatin remodeling. (B) Heatmap of C-Hi-C data showing ref6-5 specific loops (reSLs). (C) Examples of reSLs detected by C-Hi-C. C-Hi-C interaction signal (blue lines) and H3K27me3 ChIP-seq signal in wild type (black peaks) and ref6-5 (red peaks) are represented. (D) Model of chromatin contacts organization in wild type and ref6-5 mutant. (E) Histogram representing the percentage of genes (observed [O] or expected [E]) involved in reSLs that are either hyper- or hypomethylated in ref6-5 compared to WT. To obtain the expected proportion, we shuffled the H3K27me3 signals 1000 times to obtain the randomized gene counts. The mean of the 1000 permutations was used to determine the expected proportions. Asterisk indicates significant difference $\left(P\right.$-value $<2.2 \times 10^{-16}$, test of proportions). The bottom pie chart represents the percentage of ref6-5 hypermethylated genes involved in reSLs. The box plot displays the H3K27me3 levels of the $40 \%$ of ref6-5 hypermethylated genes involved on reSLs. (F) Scatterplot of $\log _{2}$ (ref6-5/wild-type gene expression fold change) for pairs of genes interacting specifically in ref6-5 compared to wild-type.

facultative heterochromatin to higher-order nuclear topology in plants is not clearly stated. In the current study, through the integration of immunostaining experiments and confocal microscopy, we provide evidence that H3K9ac-rich (euchromatin) and H3K27me3-rich (facultative heterochromatin) regions are spa- tially segregated, confirming that actively transcribed and repressed genes occupy distinct regions in the nucleus. This result is consistent with previous ChIP-based studies in Arabidopsis, which have shown that the distribution of facultative heterochromatic histone modifications such as H3K27me3 on the linear 

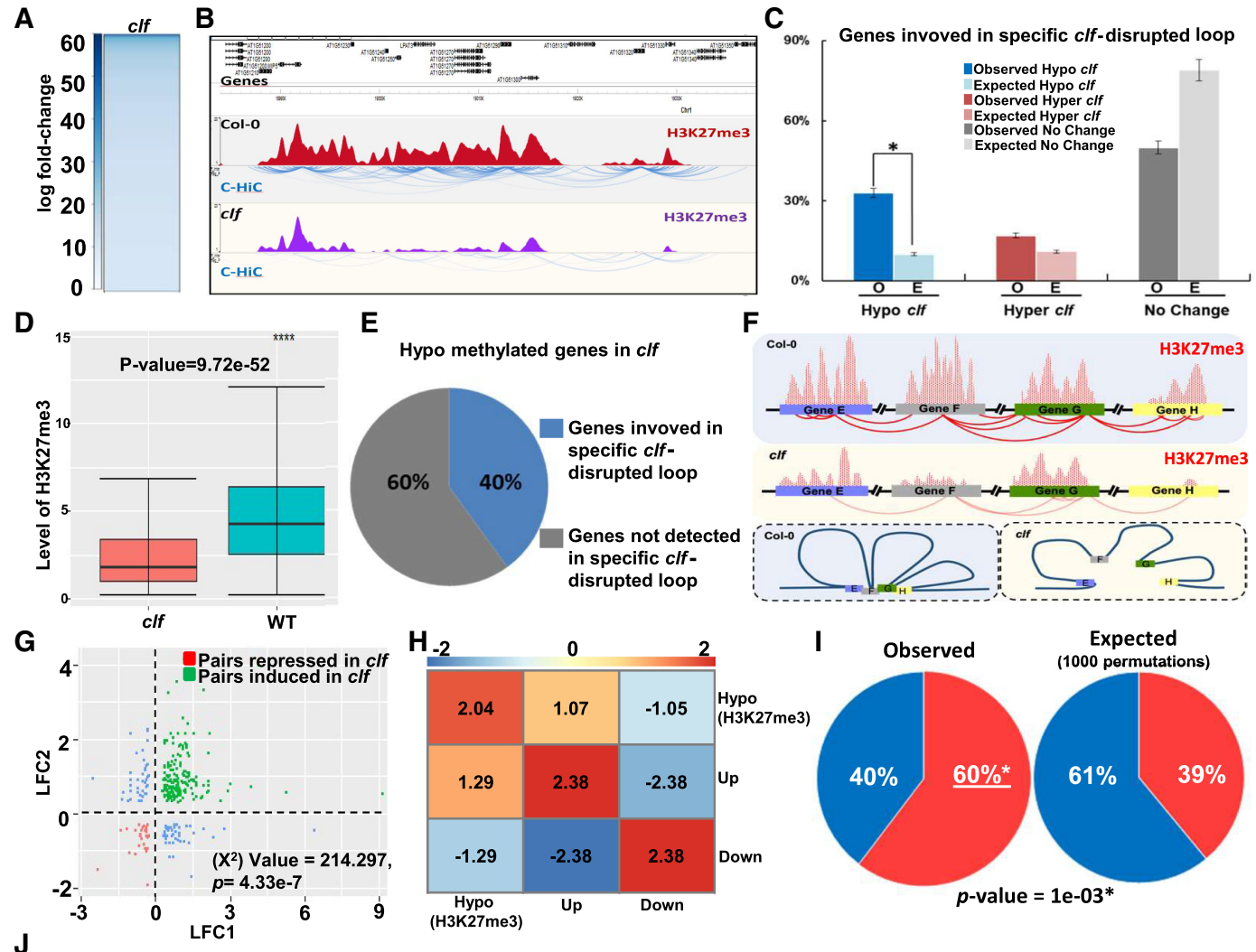

J

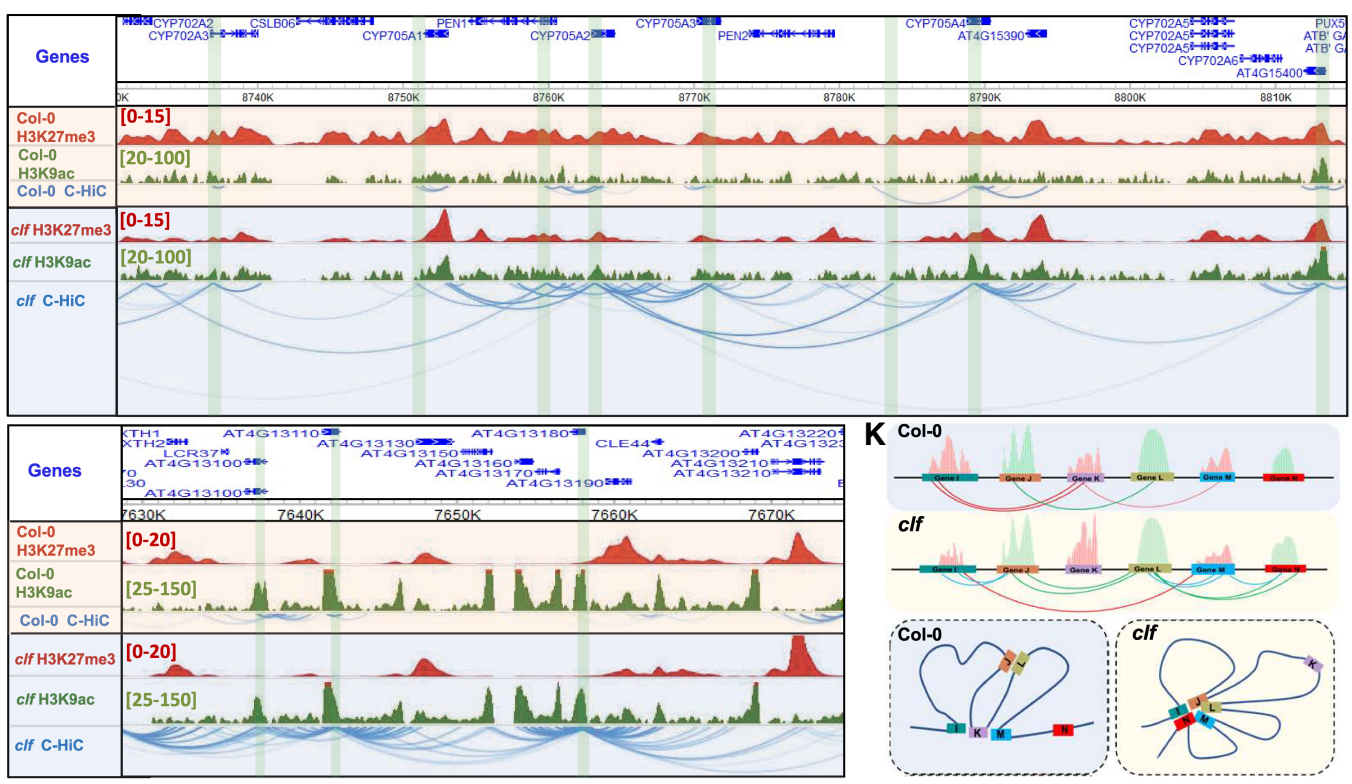

Figure 5. Reduction of $\mathrm{H} 3 \mathrm{~K} 27 \mathrm{me} 3$ levels induces a reconfiguration of chromatin architecture. ( $A$ ) Heatmap of $\mathrm{C}-\mathrm{Hi}-\mathrm{C}$ data showing loops that are weaker in clf mutant than in wild type. (B) Example of C-Hi-C interactions displaying disrupted loops in clf compared to wild type. C-Hi-C interactions (blue lines) and H3K27me3 ChIP-seq signal in wild type (red peaks) and clf (purple peaks) are represented. (C) Histogram representing the percentage of genes (observed [O] or expected [E]) involved in CSDLs that are either hyper- or hypomethylated in clf compared to WT. To obtain the expected proportion, we shuffled the H3K27me3 signals 1000 times to obtain the randomized gene counts. The mean of the 1000 permutations was used to determine the expected proportions. Asterisk indicates significant difference $\left(P\right.$-value $<2.2 \times 10^{-16}$, test of proportions). (D) The box plot displays the H3K27me3 levels of the $40 \%$ of $c l f$ hypomethylated genes involved in CSDLs. (E) The pie chart represents the percentage of clf hypomethylated genes involved in cSDLs. $(F)$ Model of chromatin contacts organization in wild type and clf mutant. $(G)$ Scatterplot of $\log _{2}$ (clf/wild-type gene expression fold change) for pairs of genes interacting specifically in wild type compared to clf. $(H)$ Heatmap presenting the $\log _{2}$ of odd ratios of combinations of features of interacting genes (Results). Positive $\log _{2}$ (odd ratio) indicates enrichment and negative indicates depletion. (I) Pie chart representing the proportion of loops involving a gene H3K27me3 hypomethylated and a gene marked or not by H3K9ac in clf mutant. (Hypo-H3K27me3) No H3K9ac loops in clf mutant (blue: 40\% observed and $61 \%$ expected, respectively); (Hypo-H3K27me3) H3K9ac loops (red: $60 \%$ observed and 39\% expected, respectively). (/) Examples of C-Hi-C interactions of a region losing $\mathrm{H} 3 \mathrm{~K} 27 \mathrm{me} 3$ in clf and that tend to establish interactions with regions marked with $\mathrm{H} 3 \mathrm{~K} 9 \mathrm{ac}$ euchromatin histone modification. $\mathrm{C}$-Hi-C interactions (blue lines), H3K9ac ChIP-seq signal in wild type and clf (green peaks), H3K27me3 ChIP-seq signal in wild type and clf (red peaks) are represented. $(K)$ Model of chromatin contacts organization in wild type and clf mutant.

\section{Genome Research}

www.genome.org 
genome is anticorrelated to euchromatic histone modifications (Ha et al. 2011).

Hi-C data is useful for the depiction of the genome-wide 3D organization of chromatin in the nucleus; however, to understand which proteins or covalent histone modifications have a role in structuring this organization, it is necessary to integrate it with external data sets (e.g., ChIP-seq) allowing only correlative analyses. To circumvent this limitation and to address whether and how genomic regions associated to histones bearing a specific covalent modification interact through long-range chromatin looping in a genome-wide and unbiased manner, we performed HiChIP experiments, confirming that transcriptionally active and inactive genes localize in different nuclear compartments. In addition, we also observed both long- and short-range interactions, ranging from a few kilobases to several megabases, enriched at the site of either H3K27me3 or H3K9ac, highlighting the role of these histone modifications in $3 \mathrm{D}$ chromatin architecture. In plants, it has been previously hypothesized that $\mathrm{H} 3 \mathrm{~K} 27 \mathrm{me} 3$ contributes to the establishment of chromatin loops, because this histone modification has been detected in interacting genomic regions (Liu et al. 2016a); however, in the current study we have taken advantage of the specific capture of H3K27me3-labeled chromatin loops to provide evidence of the role of this covalent histone modification in the spatial organization of chromatin. A similar approach has been used in maize to identify cis-regulatory elements, revealing that $\mathrm{H} 3 \mathrm{~K} 27 \mathrm{me} 3$ is potentially implicated in mediating chromatin loops between regulatory elements and their target genes in a process associated to gene repression (Ricci et al. 2019).

\section{Chromatin architecture and development}

In the last few years, we have witnessed a significant increase in studies addressing the role of 3D genome organization in the regulation of developmental processes as well as in response to environmental cues. Hence, it is currently known that chromatin topology is highly dynamic during animal developmental phase transitions, including gametogenesis, embryo development, and cell differentiation. The recent development of single-cell and low-input Hi-C approaches has permitted the characterization of the embryo along different developmental stages, as well as different cell lines during differentiation and lineage commitment. This has allowed researchers to identify commonalities and particularities of each cell type and to associate them to specific phenotypes (Zheng and Xie 2019). These single-cell techniques have seldom been applied in plants, mainly because of the technical limitations that this imposes (Huang et al. 2020); however, some studies have started characterizing the chromatin topology of specific plant cell types, showing that as in animals, gamete, zygote, and differentiated cells present striking differences in their nuclear 3D chromatin configuration. By integrating single-cell $3 \mathrm{C}$ and $\mathrm{Hi}-\mathrm{C}$ approaches, Zhou and collaborators analyzed the 3D genomes of rice sperm, egg, unicellular zygote, and mesophyll cells, finding that rice nuclei present spatially organized chromatin territories associated to different histone modifications and gene expression levels, providing evidence of the contribution of the spatial organization of chromatin to the coregulation of gene expression (Zhou et al. 2019).

The relevance of chromatin organization in the developmental context can be evidenced in the variety of diseases and developmental problems that have been associated to abnormalities in chromatin organization in various organisms. For instance, cardiac diseases, cancer and developmental disorders have been attrib- uted to mutations in genes encoding CTCFs and cohesion proteins (Lupiáñez et al. 2015, 2016; Kaiser and Semple 2017; Rosa-Garrido et al. 2017). Furthermore, the disruption of TAD boundaries has been shown to have developmental effects as a consequence of $e n$ hancer hijacking, or the abnormal interaction between an enhancer and a promoter. Several examples of this phenomenon have been reported in the literature (Lupiáñez et al. 2016; Kaiser and Semple 2017; Zheng and Xie 2019), highlighting the crucial role of chromatin organization on cell homeostasis and animal development. However, studies demonstrating the developmental consequences of chromatin disorganization in plants remain scarce. It has been recently shown that loss of CROWDED NUCLEI (CRWN) proteins, which are candidate nuclear lamina proteins in Arabidopsis, leads to a general reduction on chromatin organization and an associated reprogramming of the transcriptome (Choi et al. 2019; Hu et al. 2019). Furthermore, the loss of these proteins has been reported to induce dwarfism and a spontaneous cell death phenotype, which have been attributed to the constitutive induction of the salicylic acid-dependent pathway of immunity (Hu et al. 2019). These results suggest that as in animals, 3D chromatin organization plays a key role in the regulation of plant development and homeostasis. Furthermore, these results suggest that several of the pleiotropic phenotypes observed in plants defective in the epigenomic machinery may result from distortions in chromatin architecture and their impact on gene expression; however, this needs to be experimentally assessed.

In the current study we provide evidence for the existence of distinctive chromatin architecture between root and shoot cells, as evidenced by the presence of shoot- and root-specific repressive loops mediated by H3K27me3. This represents valuable evidence for the understanding of how chromatin modifications contribute to chromatin topology and, consequently, to organ identity in plants. Our data show that genes involved in SSRLs and RSRLs are associated with biological functions specific to the studied organs, supporting the view that chromatin loops contribute to the developmental differentiation of specific cells types in plants (Fig. 6).

\section{Polycomb-repressed chromatin is critical for 3D nuclear organization in plants}

Traditionally, Polycomb complexes have been associated to the repression of developmental genes, including the well described Hox genes (Bantignies et al. 2011; Kassis et al. 2017). However, the introduction of 3D epigenomic techniques has allowed depiction of some of the molecular mechanisms by which these complexes regulate development, including the modulation of chromatin architecture (Illingworth 2019; Pachano et al. 2019). For instance, a recent study unveiled that $\mathrm{H} 3 \mathrm{~K} 27 \mathrm{me} 3$ is a fundamental player in the chromatin reorganization occurring during oocyte development in mice (Du et al. 2020). Polycomb-associated domains (PADs) are self-interacting, cohesion-independent compartments enriched in H3K27me3 that are gradually established on the maternal genome until a late stage before disappearing upon meiotic resumption. Hi-C analyses of mutants defective on PRC2 and PRC1 subunits revealed that PAD establishment is highly dependent on these complexes and H3K27me3 (Du et al. 2020). Most of the studies on the $3 \mathrm{D}$ organization of PcG-bound chromatin in mammals have been performed in embryonic stem cells (ESCs) and have illustrated that Polycomb proteins and their associated histone modifications accumulate in ESCs in discrete foci that have been named PcG bodies (Bantignies et al. 2011; Pachano 


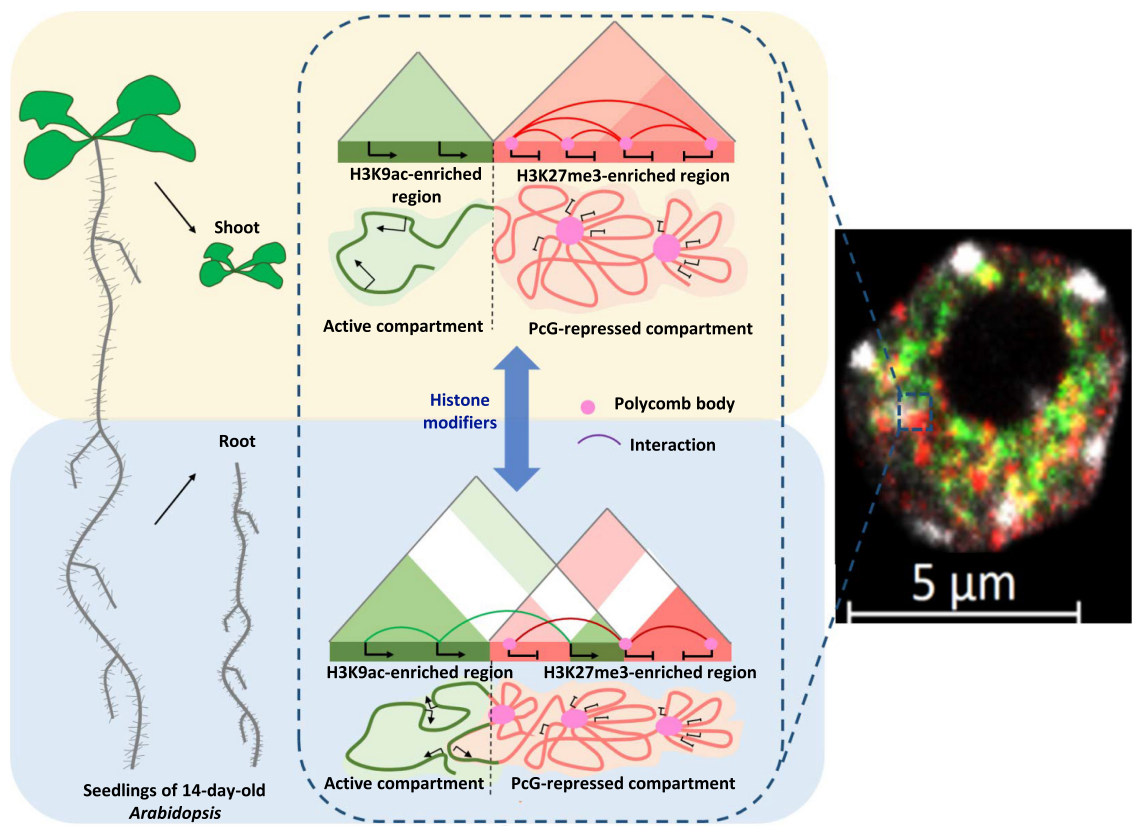

Figure 6. Histone modifiers control chromatin architecture by triggering formation of chromatin repressive or active domains to allow gene coregulation. Model of the formation of tissue-specific active and repressive chromatin domains in Arabidopsis seedlings. The green structures represent active compartments, which are associated with H3K9ac-marked euchromatin. The pink structures represent PCG-repressive compartments, which are associated with H3K27me3-marked facultative heterochromatin.

et al. 2019). Mutations in subunits of both PRC2 and PRC1 have been shown to lead to the dispersion of these structures (Hernández-Muñoz et al. 2005), suggesting significant changes in chromatin architecture in these mutants. The molecular mechanisms connecting H3K27me3 deposition, loop formation, and gene expression have been dissected into detail in Drosophila. This study showed (1) that H3K27me3 deposition is critical for loop formation, and (2) that chromatin looping contributes to gene repression, although it is not strictly required (Ogiyama et al. 2018). These results are coherent with our previous characterization of the Arabidopsis lhp1 mutant, deficient in a PRC1 subunit, where a vast proportion of genomic interactions are lost (Veluchamy et al. 2016). The loss of these interactions is associated, to a large extent, to a significant reduction on $\mathrm{H} 3 \mathrm{~K} 27 \mathrm{me} 3 \mathrm{lev}$ els on interacting partners, suggesting that both LHP1 and H3K27me3 are involved in loop formation in this organism. Because LHP1 is an H3K27me3 reader, it is considered to act, at least partially, downstream from H3K27me3 methyltransferases (Berry et al. 2017). Hence, if LHP1 activity depends on H3K27me3 levels, altering the latter through mutations on CLF or REF6 should have an impact on chromatin looping. In agreement, in the current study we found that increasing H3K27me3 levels leads to the formation of new chromatin repressive loops, whereas reducing them has the inverse effect. Altogether, these results indicate that, similar to animals, PRCs and H3K27me3 play a fundamental role in the shaping of regulatory chromatin interactions and overall, chromatin conformation in the threedimensional space, a phenomenon that is associated with developmental processes and, most likely, cell identity. However, the respective importance of $\mathrm{H} 3 \mathrm{~K} 27 \mathrm{me} 3$ deposition and H3K27me3-mediated chromatin looping for efficient gene repression will require further investigation.
We found that some of the regions that lose H3K27me3 in the clf mutant tend to establish interactions with transcribed regions marked with H3K9ac. This may indicate that one of the mechanisms by which H3K27me3 contributes to gene repression is through the preclusion of these interactions. In addition, in animals, there is recent evidence indicating that histone hyperacetylation also contributes to nuclear organization, because it induces the formation of long-range chromatin interactions and nuclear subcompartments (Rosencrance et al. 2020). This shows that the dynamic chromatin topology in the nuclear space results from the delicate balance of a plethora of elements, including various histone modifications, which recruit the nuclear machinery for its spatial organization. It has been proposed that nuclear proteins can pull the genome into new positions like in the game "cat's cradle," in which the shape of a string is successively changed by opening up new sites for finger placement (Melé and Rinn 2016). Future research is likely to provide exciting new insights into the mechanisms involving histone modifications and associated molecules in the dynamic configuration of the plant genome.

In summary, our results show the 3D organization of the genome is tightly linked to histone modifications that trigger the formation of chromatin interactions associated to gene coregulation. By these means, they contribute to proper plant development, a process in which $\mathrm{H} 3 \mathrm{~K} 27 \mathrm{me} 3$ is a key regulator of global and local facultative heterochromatin topology.

\section{Methods}

\section{Plant material and growth conditions}

Plant lines used in this study were in A. thaliana ecotype Columbia (Col-0) background. The T-DNA insertional mutants we used, clf-29 (SALK_N521003) and ref6-5 mutant (GABI_705E03), were obtained from the GABI-Kat collection (Mirouze et al. 2012). Seeds were surface-sterilized by treatment with bayrochlore, washed, and then sown in sterile half-strength MS medium, placed for $2-4 \mathrm{~d}$ at $4^{\circ} \mathrm{C}$ to obtain homogeneous germination, and plants were grown in a vertical orientation in square petri dishes in growth chambers at $20^{\circ} \mathrm{C}$ under long-day (16 h of light) conditions.

\section{Immunostaining}

Immunofluorescence labeling was performed according to Latrasse et al. (2017). Briefly, seedlings of 14-d-old Arabidopsis were fixed and then nuclei were isolated, placed on a poly-lysine slide, and incubated overnight at $4^{\circ} \mathrm{C}$ with primary antibodies specific to lysine-9-acetylated H3 (MilliporeSigma 07-352) and lysine27-trimethylated H3 (Sigma-Aldrich 07-449). After washing, slides were incubated for $1 \mathrm{~h}$ at room temperature in the dark either with Goat anti-Rabbit Alexa Fluor Plus 488 (Invitrogen A11034) and

\section{Genome Research}

www.genome.org 
Goat anti-Mouse Alexa Fluor Plus 555 (Invitrogen A32727) or with Goat anti-Rabbit Alexa Fluor Plus 555 (Invitrogen A32732) and Goat anti-Mouse Alexa Fluor Plus 488 (Invitrogen A32723) as secondary antibodies. DNA was counterstained with 4,6 diamidino-2phenylindole (DAPI) in SlowFade Diamond Antifade mounting media. Slides were directly imaged on a confocal microscope (Zeiss Microsystems).

\section{Chromatin immunoprecipitation followed by high-throughput sequencing (ChIP-seq) assay}

ChIP-seq assays were performed on shoot or root of 14-d-old seedlings using anti-H3K27me3 (Sigma-Aldrich 07-449). Plant material was cross-linked in 1\% (v/v) formaldehyde for $15 \mathrm{~min}$ at room temperature. Cross-linking was quenched with $0.125 \mathrm{M}$ glycine for $5 \mathrm{~min}$. The cross-linked plantlets were ground, and the nuclei were isolated and lysed in Nuclei Lysis Buffer (0.1\% SDS, $50 \mathrm{~mm}$ Tris- $\mathrm{HCl}$ at $\mathrm{pH} 8,10 \mathrm{~mm}$ ethylene diamine tetraacetic acid [EDTA] at pH 8). Cross-linked chromatin was sonicated for $5 \mathrm{~min}$ using a Covaris S220 (Peak Power: 175, cycles/burst: 200. Duty Factory: 20). The complexes were immunoprecipitated with antibodies, overnight at $4^{\circ} \mathrm{C}$ with gentle shaking, and incubated for $1 \mathrm{~h}$ at $4^{\circ} \mathrm{C}$ with $40 \mu \mathrm{L}$ of Dynabeads Protein A (Thermo Fisher Scientific). The beads were washed $2 \times 5 \mathrm{~min}$ in ChIP Wash Buffer 1 (0.1\% SDS, 1\% Triton X-100, $20 \mathrm{mM}$ Tris- $\mathrm{HCl}$ at $\mathrm{pH} 8$, $2 \mathrm{mM}$ EDTA at $\mathrm{pH} 8,150 \mathrm{mM} \mathrm{NaCl}), 2 \times 5 \mathrm{~min}$ in ChIP Wash Buffer 2 (0.1\% SDS, 1\% Triton X-100, $20 \mathrm{mM}$ Tris- $\mathrm{HCl}$ at $\mathrm{pH} 8$, $2 \mathrm{mM}$ EDTA at $\mathrm{pH} 8,500 \mathrm{mM} \mathrm{NaCl}), 2 \times 5 \mathrm{~min}$ in ChIP Wash Buffer 3 (0.25 mM LiCl, 1\% NP-40, 1\% sodium deoxycholate, 10 $\mathrm{mM}$ Tris- $\mathrm{HCl}$ at $\mathrm{pH} 8,1 \mathrm{mM}$ EDTA at $\mathrm{pH} 8)$ and twice in TE (10 $\mathrm{mm}$ Tris- $\mathrm{HCl}$ at $\mathrm{pH} 8,1 \mathrm{mM}$ EDTA at $\mathrm{pH} 8$ ). ChIPed DNA was eluted by two 15 -min incubations at $65^{\circ} \mathrm{C}$ with $250 \mu \mathrm{L}$ of Elution Buffer ( $1 \%$ SDS, $0.1 \mathrm{~m} \mathrm{NaHCO}_{3}$ ). Chromatin was reverse crosslinked by adding $20 \mu \mathrm{L}$ of $\mathrm{NaCl} 5 \mathrm{M}$ and incubated overnight at $65^{\circ} \mathrm{C}$. Reverse cross-linked DNA was submitted to RNase and Proteinase $\mathrm{K}$ treatment and extracted with phenol-chloroform. DNA was ethanol precipitated in the presence of $20 \mu \mathrm{g}$ of glycogen and resuspended in $10 \mu \mathrm{L}$ of nuclease-free water in a DNA low-bind tube. Libraries were then generated using $10 \mathrm{ng}$ of DNA and NEBNext Ultra II DNA Library Prep Kit for Illumina (NEB). The quality of the libraries was assessed with an Agilent 2100 Bioanalyzer (Agilent), and the libraries were subjected to $1 \times 75$ bp high-throughput sequencing by NextSeq 500 (Illumina).

\section{HiChIP assay}

HiChIP experiments were performed on shoots and roots of 14-dold seedlings using the same procedure as in Concia et al. (2020) with either anti-H3K9ac (MilliporeSigma 07-352) or antiH3K27me3 (Sigma-Aldrich 07-449). The quality of the libraries was assessed with Agilent 2100 Bioanalyzer (Agilent), and the libraries were subjected to $2 \times 75 \mathrm{bp}$ paired-end high-throughput sequencing by NextSeq 500 (Illumina).

\section{Capture Hi-C assay}

For Capture Hi-C, in situ Hi-C libraries from shoot and root of Col0, ref6, and clf were first generated as in Concia et al. (2020). The quality and the DNA concentration of the libraries were assessed with an Agilent 2100 Bioanalyzer (Agilent). Capture was then performed using SureSelect XT Target Enrichment System for Illumina Paired-End Multiplexed Sequencing Library (Agilent) according to the manufacturer's recommendation. To this end, 52,911 custom probes were designed, covering 2.658 Mbp and 4650 genes (Supplemental Table S25). Capture was performed using $1 \mu \mathrm{g}$ of in situ Hi-C libraries and following the manufacturer's recommendation. Quality control and sequencing of the libraries were performed in the same way as for HiChIP assays.

\section{Analysis of ChIP-seq data}

Single-end sequencing of ChIP samples was performed using Illumina NextSeq 500 with a read length of $76 \mathrm{bp}$. Trimmomatic- 0.38 (Martin 2011) was used for quality trimming. Parameters for read quality filtering were set as follows: minimum length of $36 \mathrm{bp}$; mean Phred quality score greater than 30; leading and trailing bases removal with base quality $<5$. The reads were mapped onto the TAIR10 assembly using Bowtie 2 (Langmead and Salzberg 2012) with mismatch permission of $1 \mathrm{bp}$. To identify significantly enriched regions, we used MACS2 (Gaspar 2018). Parameters for peaks detection were set as follows: number of duplicate reads at a location:1; mfold of 5:50; Q-value cutoff: 0.05; extsize 200; broad peak. To extract the average scores across the genomic regions, multiBigwigSummary command from the deepTools package (Ramírez et al. 2016) was used with default parameters on the RPGC normalized bigWig files.

\section{Differential expression analysis}

Single-end sequencing of RNA-seq samples were trimmed using Trimmomatic-0.38 with the following parameters: minimum length of $30 \mathrm{bp}$; mean Phred quality score greater than 30; leading and trailing bases removal with base quality $<5$. STAR aligner (Dobin et al. 2013) was used to map the reads to TAIR10 genome assembly. Raw read counts were then extracted using featureCounts based on the gene annotations in Araport11_GFF3 genes_transposons.201606.gtf. Finally, we used DESeq2 (Love et al. 2014) to identify differentially expressed genes. Genes having read counts $\geq 50$ in at least two samples were considered for differential expression analysis.

\section{Analysis of HiChIP and Capture Hi-C data}

Raw FASTQ files were preprocessed with Trimmomatic-0.38 to remove Illumina sequencing adapters. The $5^{\prime}$ and $3^{\prime}$ ends with a quality score below 5 (Phred+33) were trimmed and reads shorter than $30 \mathrm{bp}$ after trimming were dropped. The trimmed files were then processed with HiC-Pro v2.11.1 (Servant et al. 2015). The reads were aligned using Bowtie 2 onto the TAIR10 assembly with default settings, except for the parameter "--score-min L, $-0.6,-0.8$ ". Invalid ligation products (such as dangling ends, fragments ligated on themselves, and ligations of juxtaposed fragments) were discarded. Valid pairs were used to produce raw interaction matrixes at various resolutions. Finally, ".hic" files were generated with the software Juicer Tools and visualized with the tool Juicebox (Durand et al. 2016).

\section{Identification and analysis of genomic interactions}

Valid pairs generated are further analyzed using HOMER v4.10 (Zhang et al. 2008) for different resolutions (500 bp for short-range interactions and $20 \mathrm{~kb}$ for long-range interactions). For the shortrange interactions, both the anchors of the interactions (genomic bins) were annotated with genes using BEDTools intersect (Quinlan and Hall 2010). We removed interactions without any gene annotations, self-loops, and duplicates. Finally, based on the gene annotations, we integrated the log fold change from RNA-seq data and ChIP-seq data. 


\section{Data access}

All raw and processed sequencing data generated in this study have been submitted to the NCBI Gene Expression Omnibus (GEO; https://www.ncbi.nlm.nih.gov/geo/) under accession number GSE155502.

\section{Competing interest statement}

The authors declare no competing interests.

\section{Acknowledgments}

This work was supported by the Agence Nationale de la Recherche (ANR) (3DWheat project ANR-19-CE20-0001-01) and by the Institut Universitaire de France (IUF). Y.H. was supported by China Scholar Council fellowships (201806690005).

Author contributions: M.B. designed this research project. Y.H., J.S.R.-P., N.Y.R.-G., and R.B.-C. collected the plant materials. Y.H. and D.L. performed the Capture Hi-C, HiChIP, ChIP-seq experiments, and Capture Hi-C, HiChIP, ChIP-seq libraries. D.L. performed the Illumina sequencing and immunofluorescence staining experiments. S.S., J.A.-S., D.M.-M., J.A., D.L., L.C., and M.B. performed the bioinformatics analyses. Y.H., S.S., D.L., and M.B. made figures and supplemental data. M.B., F.A., and J.S.R.-P. wrote the paper with support from all authors. M.M.M., S.A., A.V.P., F.B., C.B., F.A., H.H., M.C., J.G.-M., C.R., and D.L. analyzed the data and provided critical feedback. All authors read and approved the final manuscript.

\section{References}

Ahmad A, Zhang Y, Cao X. 2010. Decoding the epigenetic language of plant development. Mol Plant 3: 719-728. doi:10.1093/mp/ssq026

Antunez-Sanchez J, Naish M, Ramirez-Prado JS, Ohno S, Huang Y, Dawson A, Opassathian K, Manza-Mianza D, Ariel F, Raynaud C, et al. 2020. A new role for histone demethylases in the maintenance of plant genome integrity. eLife 9: e58533. doi:10.7554/eLife.58533

Bantignies F, Roure V, Comet I, Leblanc B, Schuettengruber B, Bonnet J, Tixier V, Mas A, Cavalli G. 2011. Polycomb-dependent regulatory contacts between distant Hox loci in Drosophila. Cell 144: 214-226. doi:10.1016/j.cell.2010.12.026

Berry S, Rosa S, Howard M, Bühler M, Dean C. 2017. Disruption of an RNAbinding hinge region abolishes LHP1-mediated epigenetic repression. Genes Dev 31: 2115-2120. doi:10.1101/gad.305227.117

Boettiger AN, Bintu B, Moffitt JR, Wang S, Beliveau BJ, Fudenberg G, Imakaev M, Mirny LA, Wu CT, Zhuang X. 2016. Super-resolution imaging reveals distinct chromatin folding for different epigenetic states Nature 529: 418-422. doi:10.1038/nature16496

Cheng K, Xu Y, Yang C, Ouellette L, Niu L, Zhou X, Chu L, Zhuang F, Liu J, $\mathrm{Wu} \mathrm{H}$, et al. 2020. Histone tales: lysine methylation, a protagonist in Arabidopsis development. J Exp Bot 71: 793-807. doi:10.1093/jxb/ erz435

Cheutin T, Cavalli G. 2014. Polycomb silencing: from linear chromatin domains to 3D chromosome folding. Curr Opin Genet Dev 25: 30-37. doi:10.1016/j.gde.2013.11.016

Choi J, Strickler SR, Richards EJ. 2019. Loss of CRWN nuclear proteins induces cell death and salicylic acid defense signaling. Plant Physiol 179: 1315-1329. doi:10.1104/pp.18.01020

Concia L, Veluchamy A, Ramirez-Prado JS, Martin-Ramirez A, Huang Y, Perez M, Domenichini S, Rodriguez-Granados NY, Kim SK, Blein T, et al. 2020. Wheat chromatin architecture is organized in genome territories and transcription factories. Genome Biol 21: 104. doi:10.1186/ s13059-020-01998-1

Dekker J, Heard E. 2015. Structural and functional diversity of topologically associating domains. FEBS Lett 589: 2877-2884. doi:10.1016/j.febslet .2015.08.044

de Wit E, Vos ESM, Holwerda SJB, Valdes-Quezada C, Verstegen MJAM, Teunissen H, Splinter E, Wijchers PJ, Krijger PHL, de Laat W. 2015. CTCF binding polarity determines chromatin looping. Mol Cell 60: 676-684. doi:10.1016/j.molcel.2015.09.023
Dixon JR, Selvaraj S, Yue F, Kim A, Li Y, Shen Y, Hu M, Liu JS, Ren B. 2012 Topological domains in mammalian genomes identified by analysis of chromatin interactions. Nature 485: 376-380. doi:10.1038/ nature 11082

Dixon JR, Gorkin DU, Ren B. 2016. Chromatin domains: the unit of chromosome organization. Mol Cell 62: 668-680. doi:10.1016/j.molcel 2016.05.018

Dobin A, Davis CA, Schlesinger F, Drenkow J, Zaleski C, Jha S, Batut P, Chaisson M, Gingeras TR. 2013. STAR: ultrafast universal RNA-seq aligner. Bioinformatics 29: 15-21. doi:10.1093/bioinformatics/bts635

Dong P, Tu X, Chu PY, Lü P, Zhu N, Grierson D, Du B, Li P, Zhong S. 2017. 3D chromatin architecture of large plant genomes determined by local A/B compartments. Mol Plant 10: 1497-1509. doi:10.1016/j.molp.2017 .11 .005

Dong Q, Li N, Li X, Yuan Z, Xie D, Wang X, Li J, Yu Y, Wang J, Ding B, et al. 2018. Genome-wide Hi-C analysis reveals extensive hierarchical chromatin interactions in rice. Plant J 94: 1141-1156. doi:10.1111/tpj.13925

Du Z, Zheng H, Kawamura YK, Zhang K, Gassler J, Powell S, Xu Q, Lin Z, Xu $\mathrm{K}$, Zhou Q, et al. 2020. Polycomb group proteins regulate chromatin architecture in mouse oocytes and early embryos. Mol Cell 77: 825839.e7. doi:10.1016/j.molcel.2019.11.011

Durand NC, Robinson JT, Shamim MS, Machol I, Mesirov JP, Lander ES, Aiden EL. 2016. Juicebox provides a visualization system for Hi-C contact maps with unlimited zoom. Cell Syst 3: 99-101. doi:10.1016/j.cels .2015.07.012

Eichten SR, Schmitz RJ, Springer NM. 2014. Epigenetics: beyond chromatin modifications and complex genetic regulation. Plant Physiol 165: 933947. doi:10.1104/pp.113.234211

El-Sharnouby S, Fischer B, Magbanua JP, Umans B, Flower R, Choo SW, Russell S, White R. 2017. Regions of very low H3K27me3 partition the Drosophila genome into topological domains. PLoS One 12: e0172725. doi:10.1371/journal.pone.0172725

Eskeland R, Leeb M, Grimes GR, Kress C, Boyle S, Sproul D, Gilbert N, Fan Y, Skoultchi AI, Wutz A, et al. 2010. Ring1B compacts chromatin structure and represses gene expression independent of histone ubiquitination. Mol Cell 38: 452-464. doi:10.1016/j.molcel.2010.02.032

Feng S, Cokus SJ, Schubert V, Zhai J, Pellegrini M, Jacobsen SE. 2014 Genome-wide Hi-C analyses in wild-type and mutants reveal high-resolution chromatin interactions in Arabidopsis. Mol Cell 55: 694-707. doi:10.1016/j.molcel.2014.07.008

Fortin JP, Hansen KD. 2015. Reconstructing A/B compartments as revealed by $\mathrm{Hi}-\mathrm{C}$ using long-range correlations in epigenetic data. Genome Biol 16: 180 . doi:10.1186/s13059-015-0741-y

Francis NJ, Kingston RE, Woodcock CL. 2004. Chromatin compaction by a Polycomb group protein complex. Science 306: 1574-1577. doi:10 $.1126 /$ science. 1100576

Fullwood MJ, Liu MH, Pan YF, Liu J, Xu H, Mohamed YB, Orlov YL, Velkov S, Ho A, Mei PH, et al. 2009. An oestrogen-receptor- $\alpha$-bound human chromatin interactome. Nature 462: 58-64. doi:10.1038/nature08497

Gambino G, Pantaleo V. 2017. Epigenetics in plant-pathogen interactions. In Plant epigenetics (ed. Rajewsky N, et al.) pp. 385-404. Springer Nature, Cham, Switzerland.

Gaspar JM. 2018. Improved peak-calling with MACS2. bioRxiv doi:10.1101/ 496521

Gaudin V, Libault M, Pouteau S, Juul T, Zhao G, Lefebvre D, Grandjean O. 2001. Mutations in LIKE HETEROCHROMATIN PROTEIN 1 affect flowering time and plant architecture in Arabidopsis. Development 128: 48474858. doi:10.1242/dev.128.23.4847

Gonzalez-Sandoval A, Gasser SM. 2016. On TADs and LADs: spatial control over gene expression. Trends Genet 32: 485-495. doi:10.1016/j.tig.2016 .05 .004

Grob S, Schmid MW, Grossniklaus U. 2014. Hi-C analysis in Arabidopsis identifies the KNOT, a structure with similarities to the flamenco locus of Drosophila. Mol Cell 55: 678-693. doi:10.1016/j.molcel.2014.07.009

Grossniklaus U, Paro R. 2014. Transcriptional silencing by Polycomb-group proteins. Cold Spring Harb Perspect Biol 6: a019331. doi:10.1101/cshper spect.a019331

Guo Y, Xu Q, Canzio D, Shou J, Li J, Gorkin DU, Jung I, Wu H, Zhai Y, Tang $\mathrm{Y}$, et al. 2015. CRISPR inversion of CTCF sites alters genome topology and enhancer/promoter function. Cell 162: 900-910. doi:10.1016/j .cell.2015.07.038

Ha M, Ng DW, Li W, Chen ZJ. 2011. Coordinated histone modifications are associated with gene expression variation within and between species. Genome Res 21: 590-598. doi:10.1101/gr.116467.110

Hernández-Muñoz I, Taghavi P, Kuijl C, Neefjes J, van Lohuizen M. 2005 Association of BMI1 with polycomb bodies Is dynamic and requires PRC2/EZH2 and the maintenance DNA methyltransferase DNMT1. Mol Cell Biol 25: 11047-11058. doi:10.1128/MCB.25.24.11047-11058 .2005

\section{Genome Research}

www.genome.org 
Hou X, Zhou J, Liu C, Liu L, Shen L, Yu H. 2014. Nuclear factor Y-mediated H3K27me3 demethylation of the SOC1 locus orchestrates flowering responses of Arabidopsis. Nat Commun 5: 4601. doi:10.1038/ncomms5601

Hu B, Wang N, Bi X, Karaaslan ES, Weber AL, Zhu W, Berendzen KW, Liu C. 2019. Plant lamin-like proteins mediate chromatin tethering at the nuclear periphery. Genome Biol 20: 87. doi:10.1186/s13059-019-1694-3

Huang Y, Rodriguez-Granados NY, Latrasse D, Raynaud C, Benhamed M, Ramirez-Prado JS. 2020. The matrix revolutions: towards the decoding of the plant chromatin three-dimensional reality. J Exp Bot 71: 51295147. doi:10.1093/jxb/eraa322

Illingworth RS. 2019. Chromatin folding and nuclear architecture: PRC1 function in 3D. Curr Opin Genet Dev 55: 82-90. doi:10.1016/j.gde .2019 .06 .006

Joshi O, Wang SY, Kuznetsova T, Atlasi Y, Peng T, Fabre PJ, Habibi E, Shaik J, Saeed S, Handoko L, et al. 2015. Dynamic reorganization of extremely long-range promoter-promoter interactions between two states of pluripotency. Cell Stem Cell 17: 748-757. doi:10.1016/j.stem.2015.11.010

Kaiser VB, Semple CA. 2017. When TADs go bad: chromatin structure and nuclear organisation in human disease. F1000Res 6: 314. doi:10 $.12688 /$ f1000research.10792.1

Kassis JA, Kennison JA, Tamkun JW. 2017. Polycomb and trithorax group genes in Drosophila. Genetics 206: 1699-1725. doi:10.1534/genetics .115 .185116

Kouzarides T. 2007. Chromatin modifications and their function. Cell 128: 693-705. doi:10.1016/j.cell.2007.02.005

Kundu S, Ji F, Sunwoo H, Jain G, Lee JT, Sadreyev RI, Dekker J, Kingston RE. 2017. Polycomb repressive complex 1 generates discrete compacted domains that change during differentiation. Mol Cell 65: 432-446.e5. doi:10.1016/j.molcel.2017.01.009

Langmead B, Salzberg SL. 2012. Fast gapped-read alignment with Bowtie 2. Nat Methods 9: 357-359. doi:10.1038/nmeth.1923

Lanzuolo C, Roure V, Dekker J, Bantignies F, Orlando V. 2007. Polycomb response elements mediate the formation of chromosome higher-order structures in the bithorax complex. Nat Cell Biol 9: 1167-1174. doi:10 $.1038 /$ ncb1637

Latrasse D, Jégu T, Li H, de Zelicourt A, Raynaud C, Legras S, Gust A, Samajova O, Veluchamy A, Rayapuram N, et al. 2017. MAPK-triggered chromatin reprogramming by histone deacetylase in plant innate immunity. Genome Biol 18: 131. doi:10.1186/s13059-017-1261-8

Lieberman-Aiden E, van Berkum NL, Williams L, Imakaev M, Ragoczy T, Telling A, Amit I, Lajoie BR, Sabo PJ, Dorschner MO, et al. 2009. Comprehensive mapping of long-range interactions reveals folding principles of the human genome. Science 326: 289-293. doi:10.1126/sci ence.1181369

Liu C, Wang C, Wang G, Becker C, Weigel D. 2016a. Genome-wide analysis of chromatin packing in Arabidopsis thaliana at single-gene resolution. Genome Res 26: 1057-1068. doi:10.1101/gr.204032.116

Liu J, Deng S, Wang H, Ye J, Wu HW, Sun HX, Chua NH. 2016b. CURLY LEAF regulates gene sets coordinating seed size and lipid biosynthesis. Plant Physiol 171: 424-436. doi:10.1104/pp.15.01335

Liu C, Cheng YJ, Wang JW, Weigel D. 2017. Prominent topologically associated domains differentiate global chromatin packing in rice from Arabidopsis. Nat Plants 3: 742-748. doi:10.1038/s41477-017-0005-9

Love MI, Huber W, Anders S. 2014. Moderated estimation of fold change and dispersion for RNA-seq data with DESeq2. Genome Biol 15: 550 . doi:10.1186/s13059-014-0550-8

Lu F, Cui X, Zhang S, Jenuwein T, Cao X. 2011. Arabidopsis REF6 is a histone H3 lysine 27 demethylase. Nat Genet 43: 715-719. doi:10.1038/ng.854

Lupiáñez DG, Kraft K, Heinrich V, Krawitz P, Brancati F, Klopocki E, Horn D, Kayserili H, Opitz JM, Laxova R, et al. 2015. Disruptions of topological chromatin domains cause pathogenic rewiring of gene-enhancer interactions. Cell 161: 1012-1025. doi:10.1016/j.cell.2015.04.004

Lupiáñez DG, Spielmann M, Mundlos S. 2016. Breaking TADs: how alterations of chromatin domains result in disease. Trends Genet 32: 225237. doi:10.1016/j.tig.2016.01.003

Mach J. 2018. In the histone zone: the mighty eraser. Plant Cell 30: 5-6. doi:10.1105/tpc. 18.00040

Martin M. 2011. Cutadapt removes adapter sequences from high-throughput sequencing reads. EMBnet.journal 17: 10. doi:10.14806/ej.17.1.200

Mascher M, Gundlach H, Himmelbach A, Beier S, Twardziok SO, Wicker T, Radchuk V, Dockter C, Hedley PE, Russell J, et al. 2017. A chromosome conformation capture ordered sequence of the barley genome. Nature 544: 427-433. doi:10.1038/nature22043

McLaughlin K, Flyamer IM, Thomson JP, Mjoseng HK, Shukla R, Williamson I, Grimes GR, Illingworth RS, Adams IR, Pennings S, et al. 2019. DNA methylation directs polycomb-dependent 3D genome re-organization in naive pluripotency. Cell Rep 29: 1974-1985.e6. doi:10 $.1016 /$ j.celrep.2019.10.031

Melé M, Rinn JL. 2016. "Cat's cradling" the 3D genome by the act of lncRNA transcription. Mol Cell 62: 657-664. doi:10.1016/j.molcel.2016.05.011
Mifsud B, Tavares-Cadete F, Young AN, Sugar R, Schoenfelder S, Ferreira L, Wingett SW, Andrews S, Grey W, Ewels PA, et al. 2015. Mapping longrange promoter contacts in human cells with high-resolution capture Hi-C. Nat Genet 47: 598-606. doi:10.1038/ng.3286

Mirouze M, Lieberman-Lazarovich M, Aversano R, Bucher E, Nicolet J, Reinders J, Paszkowski J. 2012. Loss of DNA methylation affects the recombination landscape in Arabidopsis. Proc Natl Acad Sci 109: 58805885. doi:10.1073/pnas.1120841109

Mishra A, Hawkins RD. 2017. Three-dimensional genome architecture and emerging technologies: looping in disease. Genome Med 9: 87. doi:10 1186/s13073-017-0477-2

Misteli T. 2007. Beyond the sequence: cellular organization of genome function. Cell 128: 787-800. doi:10.1016/j.cell.2007.01.028

Mozgova I, Köhler C, Hennig L. 2015. Keeping the gate closed: functions of the polycomb repressive complex PRC2 in development. Plant J 83: 121-132. doi:10.1111/tpj.12828

Mumbach MR, Rubin AJ, Flynn RA, Dai C, Khavari PA, Greenleaf WJ, Chang HY. 2016. HiChIP: efficient and sensitive analysis of protein-directed genome architecture. Nat Methods 13: 919-922. doi:10.1038/nmeth.3999

Noh B, Lee SSH, Kim HHJ, Yi G, Shin EAEA, Lee M, Jung KJK, Doyle MMR, Amasino RMR, Noh YSY. 2004. Divergent roles of a pair of homologous jumonji/zinc-finger-class transcription factor proteins in the regulation of Arabidopsis flowering time. Plant Cell 16: 2601-2613. doi:10.1105/tpc 104.025353

Nützmann HW, Doerr D, Ramírez-Colmenero A, Sotelo-Fonseca JE, Wegel E, Di SM, Wingett SW, Fraser P, Hurst L, Fernandez-Valverde SL, et al. 2020. Active and repressed biosynthetic gene clusters have spatially distinct chromosome states. Proc Natl Acad Sci 117: 13800-13809. doi:10 $.1073 /$ pnas.1920474117

Ogiyama Y, Schuettengruber B, Papadopoulos GL, Chang JM, Cavalli G. 2018. Polycomb-dependent chromatin looping contributes to gene silencing during Drosophila development. Mol Cell 71: 73-88.e5. doi:10 .1016/j.molcel.2018.05.032

Pachano T, Crispatzu G, Rada-Iglesias A. 2019. Polycomb proteins as organizers of 3D genome architecture in embryonic stem cells. Brief Funct Genomics 18: 358-366. doi:10.1093/bfgp/elz022

Pfluger J, Wagner D. 2007. Histone modifications and dynamic regulation of genome accessibility in plants. Curr Opin Plant Biol 10: 645-652. doi:10.1016/j.pbi.2007.07.013

Quinlan AR, Hall IM. 2010. BEDTools: a flexible suite of utilities for comparing genomic features. Bioinformatics 26: 841-842. doi:10.1093/bioinfor matics/btq033

Ramírez F, Ryan DP, Grüning B, Bhardwaj V, Kilpert F, Richter AS, Heyne S, Dündar F, Manke T. 2016. deepTools2: a next generation web server for deep-sequencing data analysis. Nucleic Acids Res 44: W160-W165. doi:10.1093/nar/gkw257

Rao SSP, Huang SC, Glenn St Hilaire B, Engreitz JM, Perez EM, Kieffer-Kwon KR, Sanborn AL, Johnstone SE, Bascom GD, Bochkov ID, et al. 2017. Cohesin loss eliminates all loop domains. Cell 171: 305-320.e24. doi:10.1016/j.cell.2017.09.026

Ricci WA, Lu Z, Ji L, Marand AP, Ethridge CL, Murphy NG, Noshay JM, Galli M, Mejía-Guerra MK, Colomé-Tatché M, et al. 2019. Widespread longrange cis-regulatory elements in the maize genome. Nat Plants 5: 1237-1249. doi:10.1038/s41477-019-0547-0

Rocha PP, Raviram R, Bonneau R, Skok JA. 2015. Breaking TADs: insights into hierarchical genome organization. Epigenomics 7: 523-526. doi:10.2217/epi.15.25

Rodriguez-Granados NY, Ramirez-Prado JS, Veluchamy A, Latrasse D, Raynaud C, Crespi M, Ariel F, Benhamed M. 2016. Put your 3D glasses on: plant chromatin is on show. J Exp Bot 67: 3205-3221. doi:10 $.1093 / \mathrm{jxb} / \mathrm{erw} 168$

Rosa-Garrido M, Chapski DJ, Schmitt AD, Kimball TH, Karbassi E, Monte E, Balderas E, Pellegrini M, Shih TT, Soehalim E, et al. 2017. High-resolution mapping of chromatin conformation in cardiac myocytes reveals structural remodeling of the epigenome in heart failure. Circulation 136: 1613-1625. doi:10.1161/CIRCULATIONAHA.117.029430

Rosencrance CD, Ammouri HN, Yu Q, Ge T, Rendleman EJ, Marshall SA, Eagen KP. 2020. Chromatin hyperacetylation impacts chromosome folding by forming a nuclear subcompartment. Mol Cell 78: 112126.e12. doi:10.1016/j.molcel.2020.03.018

Rowley MJ, Corces VG. 2016. The three-dimensional genome: principles and roles of long-distance interactions. Curr Opin Cell Biol 40: 8-14. doi:10.1016/j.ceb.2016.01.009

Rowley MJ, Nichols MH, Lyu X, Ando-Kuri M, Rivera ISM, Hermetz K, Wang P, Ruan Y, Corces VG. 2017. Evolutionarily conserved principles predict 3D chromatin organization. Mol Cell 67: 837-852.e7. doi:10.1016/j molcel.2017.07.022

Schoenfelder S, Sugar R, Dimond A, Javierre BM, Armstrong H, Mifsud B, Dimitrova E, Matheson L, Tavares-Cadete F, Furlan-Magaril M, et al. 2015. Polycomb repressive complex PRC1 spatially constrains the 
mouse embryonic stem cell genome. Nat Genet 47: 1179-1186. doi:10 $.1038 / \mathrm{ng} .3393$

Servant N, Varoquaux N, Lajoie BR, Viara E, Chen CJ, Vert JP, Heard E, Dekker J, Barillot E. 2015. HiC-Pro: an optimized and flexible pipeline for Hi-C data processing. Genome Biol 16: 259. doi:10.1186/s13059. 015-0831-x

Sutherland H, Bickmore WA. 2009. Transcription factories: gene expression in unions? Nat Rev Genet 10: 457-466. doi:10.1038/nrg2592

Turck F, Roudier F, Farrona S, Martin-Magniette MLL, Guillaume E, Buisine N, Gagnot S, Martienssen RA, Coupland G, Colot V. 2007. Arabidopsis TFL2/LHP1 specifically associates with genes marked by trimethylation of histone H3 lysine 27. PLoS Genet 3: e86. doi:10.1371/journal.pgen .0030086

Veluchamy A, Jégu T, Ariel F, Latrasse D, Mariappan KG, Kim SKK, Crespi M, Hirt H, Bergounioux C, Raynaud C, et al. 2016. LHP1 regulates H3K27me3 spreading and shapes the three-dimensional conformation of the Arabidopsis genome. PLoS One 11: e0158936. doi:10.1371/journal pone.0158936

Wang C, Liu C, Roqueiro D, Grimm D, Schwab R, Becker C, Lanz C, Weigel D. 2015. Genome-wide analysis of local chromatin packing in Arabidopsis thaliana. Genome Res 25: 246-256. doi:10.1101/gr.170332 .113

Wang M, Wang P, Lin M, Ye Z, Li G, Tu L, Shen C, Li J, Yang Q, Zhang X. 2018. Evolutionary dynamics of 3D genome architecture following polyploidization in cotton. Nat Plants 4: 90-97. doi:10.1038/s41477-0170096-3
Williamson I, Eskeland R, Lettice LA, Hill AE, Boyle S, Grimes GR, Hill RE Bickmore WA. 2012. Anterior-posterior differences in HoxD chromatin topology in limb development. Development 139: 3157-3167. doi:10 1242/dev.081174

Yan W, Chen D, Smaczniak C, Engelhorn J, Liu H, Yang W, Graf A, Carles CC, Zhou DX, Kaufmann K. 2018. Dynamic and spatial restriction of Polycomb activity by plant histone demethylases. Nat Plants 4: 681689. doi:10.1038/s41477-018-0219-5

Yun M, Wu J, Workman JL, Li B. 2011. Readers of histone modifications. Cell Res 21: 564-578. doi:10.1038/cr.2011.42

Zhang X, Germann S, Blus BJ, Khorasanizadeh S, Gaudin V, Jacobsen SE. 2007. The Arabidopsis LHP1 protein colocalizes with histone H3 Lys27 trimethylation. Nat Struct Mol Biol 14: 869-871. doi:10.1038/nsmb1283

Zhang Y, Liu T, Meyer CA, Eeckhoute J, Johnson DS, Bernstein BE, Nusbaum C, Myers RM, Brown M, Li W, et al. 2008. Model-based Analysis of ChIPSeq (MACS). Genome Biol 9: R137. doi:10.1186/gb-2008-9-9-r137

Zheng H, Xie W. 2019. The role of 3D genome organization in development and cell differentiation. Nat Rev Mol Cell Biol 20: 535-550. doi:10.1038/ s41580-019-0132-4

Zhou S, Jiang W, Zhao Y, Zhou DX. 2019. Single-cell three-dimensional genome structures of rice gametes and unicellular zygotes. Nat Plants 5: 795-800. doi:10.1038/s41477-019-0471-3

Received November 6, 2020; accepted in revised form May 20, 2021.

\section{Genome Research}




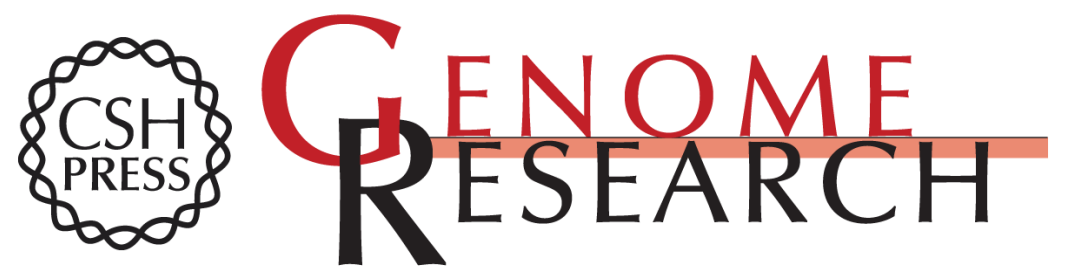

\section{Polycomb-dependent differential chromatin compartmentalization determines gene coregulation in Arabidopsis}

Ying Huang, Sanchari Sicar, Juan S. Ramirez-Prado, et al.

Genome Res. 2021 31: 1230-1244 originally published online June 3, 2021

Access the most recent version at doi:10.1101/gr.273771.120

Supplemental Material

References

Creative

Commons

License

Email Alerting

Service
http://genome.cshlp.org/content/suppl/2021/06/22/gr.273771.120.DC1

This article cites 93 articles, 20 of which can be accessed free at: http://genome.cshlp.org/content/31/7/1230.full.html\#ref-list-1

This article is distributed exclusively by Cold Spring Harbor Laboratory Press for the first six months after the full-issue publication date (see https://genome.cshlp.org/site/misc/terms.xhtml). After six months, it is available under a Creative Commons License (Attribution-NonCommercial 4.0 International), as described at http://creativecommons.org/licenses/by-nc/4.0/.

Receive free email alerts when new articles cite this article - sign up in the box at the top right corner of the article or click here.

\section{Affordable, Accurate Sequencing.}

To subscribe to Genome Research go to:

https://genome.cshlp.org/subscriptions 